\title{
Non-Coding Genetic Analysis Implicates Interleukin 18 Receptor Accessory Protein 3'UTR in Amyotrophic Lateral Sclerosis
}

\section{Authors:}

Chen Eitan ${ }^{1}$, Elad Barkan ${ }^{2}$, Tsviya Olender ${ }^{1}$, Kristel R. van Eijk ${ }^{3}$, Matthieu Moisse ${ }^{4,5}$, Sali M. K. Farhan ${ }^{6,7}$, Aviad Siany $^{1}$, Shu-Ting Hung ${ }^{8-10}$, Nancy Yacovzada ${ }^{1,2}$, Johnathan Cooper-Knock ${ }^{11}$, Kevin P. Kenna ${ }^{3}$, Rick A. A. van der Spek ${ }^{3}$, William Sproviero ${ }^{12}$, Ahmad Al Khleifat ${ }^{12}$, Alfredo Iacoangeli ${ }^{12}$, Aleksey Shatunov $^{12}$, Ashley R. Jones ${ }^{12}$, Elik Chapnik ${ }^{1}$, Daphna Rothschild ${ }^{2,13,14}$, Omer Weissbrod ${ }^{2}$, Sebastian Werneburg $^{15}$, Dorothy P. Schafer ${ }^{15}$, Robert H. Brown Jr ${ }^{16}$, Pamela J. Shaw ${ }^{11}$, Philip Van Damme ${ }^{4,5,17}$, Leonard H. van den Berg ${ }^{3}$, Hemali P. Phatnani ${ }^{18}$, Eran Segal ${ }^{2}$, Justin K. Ichida ${ }^{8-10}$, Ammar Al-Chalabi ${ }^{12,19}$, Jan H. Veldink ${ }^{3}$, Project MinE ALS Sequencing Consortium ${ }^{20}$, NYGC ALS Consortium ${ }^{20}$ and Eran Hornstein $^{1 *}$

\section{Affiliations:}

${ }^{1}$ Department of Molecular Genetics, Weizmann Institute of Science, Rehovot 7610001, Israel.

${ }^{2}$ Department of Computer Science And Applied Math, Weizmann Institute of Science, Rehovot 7610001, Israel.

${ }^{3}$ Department of Neurology, UMC Utrecht Brain Center, University Medical Center Utrecht, Utrecht University, Utrecht, 3584 CG, The Netherlands.

${ }^{4}$ KU Leuven - University of Leuven, Department of Neurosciences, Experimental Neurology, B-3000 Leuven, Belgium.

${ }^{5}$ VIB, Center for Brain \& Disease Research, Laboratory of Neurobiology, Leuven, Belgium.

${ }^{6}$ Analytic and Translational Genetics Unit, Center for Genomic Medicine, Massachusetts General Hospital and Harvard Medical School, Boston, MA 02114, USA.

${ }^{7}$ Stanley Center for Psychiatric Research, Broad Institute of MIT and Harvard, Cambridge, MA 02142, USA.

${ }^{8}$ Department of Stem Cell Biology and Regenerative Medicine, Keck School of Medicine, University of Southern California, Los Angeles, CA 90033, USA.

${ }^{9}$ Eli and Edythe Broad CIRM Center for Regenerative Medicine and Stem Cell Research at USC, Los Angeles, CA 90033, USA.

${ }^{10}$ Zilkha Neurogenetic Institute, Keck School of Medicine of the University of Southern California, Los Angeles, CA 90033, USA. 
${ }^{11}$ Sheffield Institute for Translational Neuroscience (SITraN), University of Sheffield, Sheffield S10 2HQ, UK.

${ }^{12}$ King's College London, Maurice Wohl Clinical Neuroscience Institute, Institute of Psychiatry, Psychology \& Neuroscience, De Crespigny Park, London, SE5 8AF, United Kingdom.

${ }^{13}$ Department of Developmental Biology, Stanford University, Stanford, CA 94305, USA ${ }^{14}$ Department of Genetics, Stanford University, Stanford, CA 94305, USA

${ }^{15}$ Department of Neurobiology, Brudnick Neuropsychiatric Research Institute, University of Massachusetts Medical School, Worcester, MA 01605, USA.

${ }^{16}$ Department of Neurology, University of Massachusetts Medical School, Worcester, MA 01655, USA.

${ }^{17}$ University Hospitals Leuven, Department of Neurology, Leuven, Belgium.

${ }^{18}$ Center for Genomics of Neurodegenerative Disease, New York Genome Center.

${ }^{19}$ King's College Hospital, Denmark Hill, London, SE5 9RS, United Kingdom.

${ }^{20} \mathrm{~A}$ list of Consortiums PIs and affiliations appears in the Supplementary Information.

*Corresponding author. Tel: $\quad+972 \quad 89346215 ; \quad$ Fax: $+972 \quad 89342108$; E-mail: Eran.hornstein@weizmann.ac.il 


\begin{abstract}
:
The non-coding genome is substantially larger than the protein-coding genome, but the lack of appropriate methodologies for identifying functional variants limits genetic association studies. Here, we developed analytical tools to identify rare variants in pre-miRNAs, miRNA recognition elements in 3'UTRs, and miRNA-target networks. Region-based burden analysis of $>23,000$ variants in 6,139 amyotrophic lateral sclerosis (ALS) whole-genomes and 70,403 non-ALS controls identified Interleukin-18 Receptor Accessory Protein (IL18RAP) 3'UTR variants significantly enriched in non-ALS genomes, replicate in an independent cohort and associate with a five-fold reduced risk of developing ALS. IL18RAP 3'UTR variants modify $\mathrm{NF}-\kappa \mathrm{B}$ signaling, provide survival advantage for cultured ALS motor neurons and ALS patients, and reveal direct genetic evidence and therapeutic targets for neuro-inflammation. This systematic analysis of the non-coding genome and specifically miRNA-networks will increase the power of genetic association studies and uncover mechanisms of neurodegeneration.
\end{abstract}


bioRxiv preprint doi: https://doi.org/10.1101/2021.06.03.446863; this version posted June 5, 2021. The copyright holder for this preprint (which was not certified by peer review) is the author/funder. All rights reserved. No reuse allowed without permission.

One Sentence Summary: Non-coding genetics demonstrate rare variants in IL-18 receptor 3'UTR that modifies ALS risk and progression. 


\section{Introduction}

3 Genomic sequencing technologies facilitate identification of variants in open reading frames (ORFs). 4 Although allelic variants in non-coding regions are expected to be numerous ${ }^{1,2}$ they are largely 5 overlooked because current analytical approaches do not adequately prioritize variants that are likely to 6 be functional, over neutral background variation.

7 Amyotrophic lateral sclerosis (ALS) is a fatal neurodegenerative syndrome, primarily affecting the human 8 motor neuron system with a strong genetic predisposing component ${ }^{3,4}$. Thus, mutations in approximately 925 protein-coding genes have been associated with $\mathrm{ALS}^{3,5,6}$ and a hexanucleotide repeat expansion in an 10 intronic sequence of the C9orf72 gene is the most common genetic cause of ALS ${ }^{7-9}$. However, non-coding nucleotide variants in ALS have yet to be systematically explored.

MicroRNAs (miRNAs) are endogenous posttranscriptional repressors that silence mRNA expression through sequence complementarity. miRNA dysregulation has been implicated in ALS pathogenesis, and ALS-associated RNA-binding proteins, TARDBP/TDP-43 and FUS, regulate miRNA biogenesis ${ }^{10-21}$. miRNA primarily act on $3^{\prime}$ untranslated regions (3'UTRs) ${ }^{22}$, which are non-coding parts of messenger RNAs (mRNAs) and often regulate degradation and translation ${ }^{23}$.

Burden analysis is a genetics approach that is based on the rationale that different rare variants in the same gene may have a cumulative contribution ${ }^{24}$. Therefore, burden analysis allows the identification of genes containing an excess of rare and presumably functional variation in cases relative to controls. Although de novo mutations in non-coding regions were recently shown in family-based autism studies ${ }^{25}$, variants in non-coding regions are not routinely included in rare-variant burden association studies. The application

22 of burden analysis to non-coding regulatory variation is constrained by the availability of whole genome sequencing (WGS) data, and the ability to recognize functional variants in non-coding regulatory regions, which is currently far less effective than for protein-coding genes.

To effectively apply burden analyses to regulatory variation, appropriate ways to determine what are the relevant (qualifying) variants should be determined. In the case of miRNAs and miRNA recognition elements such a framework can be achieved with relatively high confidence because of miRNA high 
28 conservation and wealth of mechanistic insight about sequence impact on function. Therefore, miRNA 29 regulatory networks make an excellent gene set to explore.

30 Here, we developed tools that identify, or call, qualifying variants in miRNAs and 3'UTR of mRNAs, and 31 performed collapsed genetic analysis ${ }^{26}$, to test if these regulatory RNAs are associated with ALS. We 32 discovered an enrichment of rare variants in the IL18RAP 3'UTR, implicating the IL-18 pathway in ALS. 33 Non-coding variants analysis in miRNA networks may impact research of human traits, increase the power 34 of rare-variant association methods and encourage systematic exploration of non-coding regions, to 35 uncover genetic mechanisms of disease. 


\section{Results}

To test whether genetic variations in non-coding regulatory regions are associated with ALS, we analyzed regions of interest WGS data from the Project MinE ALS sequencing consortium ${ }^{27}$ (Supplementary Fig. 1A,B and Supplemental Tables 1,2). The discovery cohort consisted of 3,955 ALS patients and 1,819 ageand sex-matched controls, for a total of 5,774 whole-genomes from the Netherlands, Belgium, Ireland, Spain, United Kingdom, United States and Turkey (Project MinE Datafreeze 1). We tested 295 genes, including candidates from sporadic ALS GWAS ${ }^{28}$ or encoding RNA-binding proteins, and analyzed both their 3'UTRs and open reading frames (Supplementary Table 3). In addition, we tested all 1,750 human precursor miRNA genes (pre-miRNAs; miRBase v20) ${ }^{29}$.

We devised a method for identifying rare genetic variants with minor allele frequencies (MAF) $\leq 0.01$, in non-coding RNA regulation that: (1) abrogate miRNA recognition elements in 3'UTRs; or (2) result in a predicted de novo gain of miRNA binding; or (3) change the sequence of mature miRNAs and/or premiRNAs (miRBase $\mathrm{v} 20^{29}$ ). We performed region-based burden test, in which variants within miRNA recognition elements in 3'UTRs and within pre-miRNA genes that satisfy above criteria (qualifying variants), were binned together to weight their contribution to disease.

We also identified rare variants in open reading frame of the 295 miRNA-relevant or ALS-relevant protein coding genes, that are predicted to cause frameshifting, alternative splicing, an abnormal stop codon, or a deleterious non-synonymous amino acid substitution that were detected in $\geq 3$ of 7 independent dbNSFP prediction algorithms ${ }^{30}$ (Fig. 1A and Supplementary Table 3). In total 28,211 rare qualifying variants were identified (Supplementary Table 4).

As positive control we performed an association analysis of rare variants in protein-coding sequences, using the Optimized Sequence Kernel Association Test (SKAT-O) ${ }^{31}$. SKAT-O identified a significant excess of deleterious minor alleles in the ALS genes NEK1 (127 cases; 19 controls [3.21\%; 1.04\%]: P = $\left.7.04 \times 10^{-7} ; P_{\text {corrected }}=2.13 \times 10^{-4}\right)$, comparable with a reported prevalence of $3 \%{ }^{32}$, and SODI (36 cases [0.91\%]; 0 controls: $\left.\mathrm{P}=2.61 \times 10^{-4} ; P_{\text {corrected }}=3.76 \times 10^{-2}\right)^{33}$, which is below the reported $2 \%$ prevalence ${ }^{5}$, 34 (Fig. 1B, Supplementary Fig. 2A and Data File S1). Other known ALS genes did not reach statistical significance (Supplementary Table 3), consistent with reported statistical power limitations of Project MinE WGS data in assessing the burden of rare variants ${ }^{35}$. Our analysis did not consider C9orf72 hexanucleotide (GGGGCC) repeat expansion region. 
We determined the burden of rare variants in all autosomal pre-miRNAs in the human genome $(1,750$ genes). We did not identify disease association for any pre-miRNAs, nor for any of the predicted genetic networks based on variants aggregated over specific mature miRNAs and their cognate downstream 3'UTR targets. This may be because the small size of miRNA genes makes genetic aggregation studies particularly challenging (Supplementary Fig. 2B,C).

Finally, we tested the burden of variants that are potentially either abrogating conserved miRNA binding sites or creating new miRNA binding sites in 3'UTRs. The strongest association was for the 3'UTR of IL18RAP (Fig. 1B, Supplementary Fig. 2D and Data File S1). This association was higher than expected at random $\left(\mathrm{P}=3.34 \times 10^{-5}, P_{\text {corrected }}=9.31 \times 10^{-3}\right)$ and from the association gained for all protein-coding ALS genes in this cohort, with the exception of NEK1. Notably, the signal was more prevalent in controls $[9 / 1819,0.49 \%]$ relative to ALS patients [4/3955, 0.10\%], indicating that these variants are protective against ALS.

Because the number of ALS genomes was 2.17 -fold larger than the number of controls, the data depict a 4.89-fold enrichment in the abundance of variants in controls over cases. IL18RAP 3'UTR protective variants reduced the disease odds ratio by five-fold $(\mathrm{OR}=0.20$; Fig. $2 \mathrm{~A})$, and was consistent across independent population strata (Fig. 2B), whereas $N E K 1$ and SOD1 increased the disease odds ratio $(\mathrm{OR}=$ 3.14, 33.89, respectively; Fig. 2A).

ILI8RAP 3'UTR also ranked as the top hit when we relaxed the analysis by including all 3'UTR variants, regardless of predicted miRNA recognition elements. Therefore, the robust association of ILI8RAP $3^{\prime} \mathrm{UTR}$ is independent of the assumptions about specific miRNA binding (SKAT-O P $=1.88 \times 10^{-5}, \mathrm{P}_{\text {corrected }}$ $=5.62 \times 10^{-3}$; variant in controls [12/1819, 0.66\%], cases [6/3955, 0.15\%], OR =0.23; Fig. 2A,C, Fig. 3A, Supplementary Table 5 and Data File S1). Three other algorithms - the Sequence Kernel Association Test (SKAT, $\mathrm{P}=1.73 \times 10^{-5}$; permutated $\mathrm{P}$-value $<10^{-4}$ ), the Combined Multivariate and Collapsing $(\mathrm{CMC}, \mathrm{P}$ $=8.66 \times 10^{-4}$ ) or Variable Threshold (VT) with permutation analysis (permutated P-value $=2.10 \times 10^{-3}$ ) all ranked the ILI8RAP 3'UTR association above any other studied 3'UTR, suggesting that the association does not depend on a particular statistical genetics method (Supplementary Fig. 3). Together, rare variants in IL18RAP 3'UTR are depleted in ALS patients, suggesting that they are protective against ALS.

To determine if the rare ILI8RAP 3'UTR variants are depleted in another ALS cohort, we performed independent replication studies. Similar results for rare IL18RAP 3'UTR variants were reproduced in the 
New York Genome Center (NYGC) ALS Consortium cohort (2,184 ALS genomes), which was studied against: (i) 263 non-neurological controls from the NYGC; (ii) 62,784 non-ALS genomes from NHLBI's Trans-Omics for Precision Medicine (TOPMed); and (iii) 5,537 non-ALS genomes from gnomAD. This replication effort yielded a joint analysis $\mathrm{P}$-value $=9.58 \times 10^{-4}(\chi 2$ with Yate's correction; OR $=0.32 ; 95 \%$ CI: 0.16 - 0.64; Fig. 2C and Supplementary Table 6). Combining this cohort with our discovery cohort from Project MinE, yielded a superior joint P-value < $1.00 \times 10^{-5}(\chi 2$ with Yate's correction; OR=0.20; 95\% CI: 0.12 - 0.34; Fig. 2C). A meta-analysis of Project MinE datafreeze 1 and 2, which consisted of 5,185 ALS patients and 2,262 age- and sex-matched controls, reproduced the initial signal $\left(\mathrm{p}=7.6 \times 10^{-4}\right)$.

Together, IL18RAP 3'UTR sequence variants contribute to a lower risk of suffering from ALS, that is approximately one fifth of the general population, although it did not reach conventional exome-wide multiplicity-adjusted significance threshold $\left(\alpha \approx 2.6 \times 10^{-6}\right.$, ref. $\left.{ }^{24}\right)$ in our study.

Finally, genome-wide analysis of all known human 3'UTRs (RefSeq, ${ }^{36}$ ) identified IL18RAP 3'UTR as the most significant 3'UTR associated with ALS, in the Project MinE cohort (Fig. 3B), followed by the GPATCH8 3'UTR (SKAT-O P = 1.92 $\times 10^{-5}, \mathrm{P}_{\text {corrected }}=0.16$; variant in controls [63/1819, 3.46\%], cases $[68 / 3955,1.72 \%], \mathrm{OR}=0.49)$, the $C D C 14 B$ 3'UTR (SKAT-O P = 3.64x 10 ${ }^{-5}, \mathrm{P}$ corrected $=0.19$; variant in controls [112/1819,6.16\%], cases [163/3955, 4.12\%], OR =0.66), and the RAB3GAP2 3'UTR (SKAT-O $\mathrm{P}=4.51 \times 10^{-5}, \mathrm{P}_{\text {corrected }}=0.19$, variant in controls $[147 / 1819,8.08 \%]$, cases $[485 / 3955,12.26 \%], \mathrm{OR}=$ 1.59). GPATCH8 is involved in hyperuricemia pathophysiology, $C D C 14 B$ is a dual-specificity phosphatase involved in the DNA damage response, and RAB3GAP2 is involved in neurotransmitter and hormone exocytosis and highly expressed in the brain, however, their potential role in neurodegeneration is unknown.

To investigate the source of the signal in the ILI8RAP 3'UTR in a post-hoc analysis, we divided the 11 116 patients) or two variants that were identified solely in controls ( 2 variants / 9 controls / 0 patients). While the signal of the nine singleton variants was not statistically significant, analysis of the two control variants, which were identified in multiple samples, derived an improved significance compared to the original signal $\left(\mathrm{SKAT}-\mathrm{O} \mathrm{P}=4.36 \times 10^{-6}\right)$. Thus, these two rare variants $(\mathrm{V} 1, \mathrm{Chr} 2: 103068691 \mathrm{C}>\mathrm{T}$; V3, Chr2:103068718 G>A) are likely central in generating the genetic association signal in IL18RAP 3'UTR. 
IL18RAP is a receptor subunit which dimerizes with IL18R1 upon binding of the interleukin IL-18. IL18 receptor is expressed in T-cells, neurons, astrocytes, and microglia ${ }^{37}$ and induces NF- $\kappa \mathrm{B}$ signaling. To determine the functional impact of the ILI8RAP variants we analyzed ILI8RAP expression in lymphoblastoid cell lines (LCLs) from the UK MNDA DNA Bank ${ }^{38}$ that were originally derived from two different individuals, one carrying the putative IL18RAP protective variant (V3, Chr2:103068718 G>A) and the other carrying the canonical IL18RAP 3'UTR (control). LCLs harboring the IL18RAP 3'UTR protective variant significantly down-regulated IL18RAP protein expression (Fig. 4A and Data File S2), with p-NF-кB protein levels also being significantly reduced (Fig. 4B and Data File S3). Therefore, a variant form of ILI8RAP 3'UTR attenuates its endogenous expression and downstream NF$\kappa \mathrm{B}$ signaling.

To further establish the functional relevance of the IL18RAP 3'UTR variants, we subcloned wild-type IL18RAP 3'UTR (WT) and the two most prevalent 3'UTR variants, (Chr2:103068691 C>T (V1) and Chr2:103068718 G>A (V3)), downstream of a Renilla luciferase reporter (hRluc). Variants V3 and V1 reduced luciferase activity by $\sim 33 \%$ and $\sim 30 \%$, respectively, relative to the WT ILI8RAP 3'UTR (Fig. 4C,D). Thus, the protective ILI8RAP variants regulate ILI8RAP mRNA expression.

To determine the ability of the IL18RAP variants V3 and V1 to induce NF- $\kappa \mathrm{B}$ activity, we co-transfected U2OS cells with different IL18RAP coding region (CDS) and 3'UTR constructs, along with an NF- $\mathrm{B}$ activity reporter that drives luciferase (Luc2P) transcription via five copies of the NF- $\kappa \mathrm{B}$ response element. NF- $\kappa \mathrm{B}$ signaling was induced by adding a human recombinant IL-18 to the medium. Variants $\mathrm{V} 3$ and V1 of the IL18RAP 3'UTR reduced NF- $\kappa \mathrm{B}$ activity by $\sim 10 \%$ and $\sim 21 \%$, respectively, relative to the WT IL18RAP 3'UTR (Fig. 4E,F). GFP vector and a dominant negative coding mutant E210A-Y212AY214A CDS + WT 3'UTR (3CDS) ${ }^{39}$, served as controls. We conclude that V3 and V1 3'UTR variants affect IL18RAP capacity to induce NF- $\kappa$ B signaling, although physiological relevance cannot be elucidated from a reporter assay.

Levels of the cytokine IL-18 are elevated in ALS patient tissues and biofluids ${ }^{40-42}$, but the expression in motor neurons is not characterized. To study IL-18 and ILI8RAP expression levels specifically in human motor neurons of patients with ALS, we mined human NGS data. ILI8RAP and IL-18 mRNA expression are higher in laser capture microdissection- enriched surviving motor neurons from lumbar spinal cords of patients with sALS with rostral onset and caudal progression, relative to non-neurodegeneration controls (Supplementary Fig. 4A,B; data from ref. ${ }^{43}$ ). Consistently, higher $I L-18 \mathrm{mRNA}$ levels were also 
152 in induced human motor neurons of patients with ALS (Supplementary Fig. 4C; data from ref. ${ }^{44}$ )). Thus, $153 I L-18$ and ILI8RAP receptor subunit are abnormally high in human ALS motor neurons.

154 To study the impact of ILI8RAP 3'UTR mutations in a model of human ALS motor neurons, we 155 performed a survival analyses using induced pluripotent stem cells (iPSCs) derived from human ALS patients that harbor a $C 9$ orf72 hexanucleotide repeat expansion ${ }^{45}$. iPSCs from patients and from healthy controls were differentiated to Hb9::RFP+ human motor neurons (iMNs) ${ }^{45}$ and time-lapse microscopy was used to quantify their subsequent survival after withdrawal of neurotrophic factors and in the presence of the cytokine IL-18 (Fig. 5A). As expected, degeneration (cellular death) was significantly more severe in C9orf72 patient iMNs than in iMNs derived from healthy controls $(\mathrm{n}=2$ patients, $\mathrm{n}=2$ controls, iMNs from each genotype combined into a single trace for clarity; Fig. 5B). Notably, this was partially rescued by transducing C9orf72 iPSCs with the IL18RAP 3'UTR variant V3 (including CDS) expression vector, which ameliorated motor neuron toxicity, relative to wild-type IL18RAP 3'UTR. This was similar to the effect of a IL18RAP dominant negative coding mutant, 3CDS (E210A-Y212A-Y214A; one-sided logrank test, $\mathrm{P}<0.05, \mathrm{n}=2$ patients, $\mathrm{n}=2$ controls, Fig. 5B-D). The V1 variant had a more limited effect, which might be related to activity of V1 in cells other than motor neurons. Additionally, C9orf72 iMNs harboring the V3 variant displayed a lower hazard ratio (cellular death propensity) than C9orf72 iMNs harboring the wild-type IL18RAP 3'UTR, (n=2 patients, n=2 controls (Fig. 5E). Thus, both IL18RAP and its ligand IL-18 are upregulated in human ALS motor neurons and a rare variant of IL18RAP 3'UTR confers protection to human $C 9$ orf72 motor neurons in tissue culture.

To determine whether the mutant ILI8RAP 3'UTR is also protective in human patients with ALS, we tested the association between age of diagnosis and age of death in ALS patients harboring wild-type or variants of the ILI8RAP 3'UTR. Of 4216 patients for whom data on age of diagnosis was available (Project MinE and NYGC cohorts), 8 harbored IL18RAP 3'UTR variants. Of 4263 patients for whom age of death was available, 9 harbored IL18RAP 3'UTR variants. IL18RAP variants are expected to be depleted in ALS genomes, nonetheless in those extremely rare patients harboring IL18RAP 3'UTR variants, these were associated with an older age of death, on average 6.1 years after the average for patients with canonical Il18RAP 3'UTR (one-sided Mann-Whitney test $\mathrm{P}=0.037$; Fig. 5F), and an older age of diagnosis, on average 6.2 years after the average for patients with canonical Il18RAP 3'UTR (onesided Mann-Whitney test $\mathrm{P}=0.06$; Fig. 5G). Thus, variants in IL18RAP 3'UTR are protective against ALS and provide survival advantage for patients suffering from the disease. 


\section{Discussion}

Data from the ALS consortia used in this study, Project MinE and NYGC, provide unprecedented opportunities for investigating the role of the non-coding genome in ALS, and will drive the development of computational methodologies for weighting the effect of variants outside of protein open reading frames. By identifying qualifying variants and performing rare variant aggregation analysis in 1,750 premiRNA genes, and 295 protein coding ORFs and their 3'UTRs, we demonstrated that variants in the 3'UTR of IL18RAP are enriched in non-ALS genomes, indicating that these are relatively depleted in ALS. ILI8RAP 3 'UTR variants reduced the chance of the disease five-fold, increased the survival of human C9orf72 motor neurons and delayed onset and therefore age of death in people with ALS. The discovery of functional, disease-modifying ILI8RAP 3'UTR variants underscores the need to explore the role of additional non-coding genomic regions in ALS.

Protective protein-coding variants have been identified in Alzheimer's disease ${ }^{46-49}$ and implicated in ALS as well ${ }^{50,51}$ and deleterious variants were suggested in VEGF promoter/5'UTR ${ }^{52}$. However, the $3^{\prime} \mathrm{UTR}$ of ILI8RAP is the first protective non-coding allele associated with a neurodegenerative disease.

Neuro-inflammation is prevalent in neurodegeneration, including in ALS ${ }^{53}$, and is often characterized by the activation of microglia, astrocytes, and the accumulation of infiltrating T-cells at sites of neurodegeneration ${ }^{54-57}$. The soluble ligand, IL-18, is part of this neuro-inflammatory milieu, promoting receptor subunit (IL18RAP, IL18R1) dimerization on the membrane of T-cells, neurons, astrocytes, and microglia ${ }^{37}$, and activating intracellular signaling cascades, including NF- $\kappa$ B. Polymorphic forms of IL18RAP are genetically associated with autoimmune / inflammatory diseases 58-62, suggesting that perhaps changes to IL18RAP via its 3'UTR, alter ALS risk or severity in a dose or expression-dependent manner.

Our study directly links dysregulation of IL18RAP signaling to ALS. The protective effect of IL18RAP 3'UTR variants causes downregulation of IL-18 signaling, which might be hyperactive in ALS motor neurons and is supported by previous observations showing that ALS patients have elevated levels of the cytokine IL-18 in tissues and biofluids ${ }^{40-42}$ and that IL-18 secretion is triggered from microglia in a model of TDP-43 proteinopathy ${ }^{63}$. Our data from human motor neurons further suggest that IL18RAP may be acting directly on motor neurons. Therefore, variants in IL18RAP 3'UTR may modify IL-18 signaling in 
213 the central nervous system of ALS patients. However, the regulatory changes affected by the ILI8RAP 214 3'UTR variants remains to be elucidated.

216 A limitation of our study is that only 295 candidate genes were initially tested. However, the key findings 217 were reproduced in a genome-wide study of all human 3'UTRs. While IL18RAP 3'UTR signal is 218 comparable to that of protein-coding ALS-causing genes, such as SOD1 and NEK1, limitations in the 219 statistical power may be overcome with larger ALS and control cohorts, which are not currently available. 220 Furthermore, the genetic involvement of IL18RAP 3'UTR in other neurodegenerative diseases remains to 221 be explored. Finally, the mechanism underlying IL18RAP dose sensitivity is not fully understood. While 222 we provide evidence that ILI8RAP 3'UTR endows neuroprotection to human motor neurons and is 223 associated with survival advantage in humans with ALS, additional studies should explore the mechanism 224 by which IL18RAP protects motor neurons and the degree to which other cell types, such has microglia, 225 are involved.

227 In summary, we have identified the ILI8RAP 3'UTR as a non-coding genetic disease modifier by burden 228 analysis of WGS data using ALS case-control cohorts. We show that IL-18 signaling modifies ALS 229 susceptibility and progression, delineating a neuro-protective pathway and identifying potential 230 therapeutic targets for ALS. Whereas the 3'UTR of ILI8RAP is the first protective non-coding allele 231 associated with a neurodegenerative disease, the increasing wealth of WGS data in Project MinE, NYGC 232 and elsewhere, indicates that the exploration of non-coding regulatory genomic regions should reveal 233 further disease-relevant genetic mechanisms. 


\section{Methods}

\section{Human genetic cohorts}

All participants contributed DNA after signing informed consent at the submitting sites. Human materials were studied under approval of the Weizmann Institute of Science Institutional Review Board (Weizmann IRB: 1039-1).

Discovery cohort: Project MinE ALS sequencing consortium Datafreeze 1 includes 3,955 ALS patients and 1,819 age- and sex-matched controls, free of any neurodegenerative disease, for a total of 5,774 quality control (QC) passing whole-genomes, from the Netherlands, Belgium, Ireland, Spain, United Kingdom, United States and Turkey. Rare variant association in cases versus controls was evaluated for regions of interest, when we could identify $\geq 2$ variants per region, by SKAT-O, SKAT, CMC and VT in RVTESTS environment ${ }^{64}$, with sex and the top 10 principal components (PCs) as covariates. To construct the PCs of the population structure, an independent set of $\sim 450,000$ SNPs was sampled from WGS, (MAF $\geq 0.5 \%$ ) followed by LD-pruning.

Replication cohorts: Utilized for testing rare variant alleles (MAF < 0.01) in human IL18RAP 3'UTR (GRCh37/hg19 chr2:103068641-103069025 or GRCh38 chr2:102452181-102452565) from Project MinE datafreeze 2: 1300 European heritage ALS genomes without middle eastern (Turkish and Israelis) genomes. The New York Genome Center (NYGC) ALS Consortium (2,184 ALS Spectrum MND and 263 non-neurological control genomes from European/Americas ancestries), NHLBI's Trans-Omics for Precision Medicine (TOPMed; 62,784 non-ALS genomes) and gnomAD (5,537 non-ALS genomes; Europeans, non-Finnish, non-TOPMed). Joint analysis in replication cohort, was performed by Chi square test with Yate's correction. Meta-analysis was not possible because TOPMed and gnomAD covariate information is not available.

\section{Quality control procedures in Project MinE genomics}

Sample selection, data merging and sample- and variant level quality control procedures for Project MinE ALS sequencing consortium genomes are described in full previously ${ }^{35}$. Briefly, 6,579 Project MinE ALS sequencing consortium whole genomes sequenced on Illumina HiSeq2000 or HiSeqX platforms. Reads were aligned to human genome build hg19 and sequence variants called with Isaac Genome Alignment 
262

263

264

265

266

267

268

269

270

271

272

273

274

275

276

277

278

279

280

281

282

283

284

285

286

287

288

Software and variant caller ${ }^{65}$. Individual genomic variant call format files (GVCFs) were merged with 'agg' tool: a utility for aggregating Illumina-style GVCFs. Following completion of the raw data merge, multiple QC filtering steps were performed: (i) setting genotypes with GQ<10 to missing; (ii) removing low-quality sites (QUAL $<30$ and QUAL $<20$ for SNPs and indels, respectively); (iii) removing sites with missingness $>10 \%$. (iv) Samples excluded if deviated from mean by more than 6SD for total numbers of SNPs, singletons and indels, Ti/Tv ratio, het/hom-non-ref ratio and inbreeding (by cohort). (v) missingness $>5 \%$, (vi) genotyping-sequence concordance (made possible by genotyping data generated on the Illumina Omni 2.5M SNP array for all samples; 96\% concordance), (vii) depth of coverage, (viii) a gender check (to identify mismatches), (ix) relatedness (drop samples with >100 relatedness pairs). (x) Related individuals were further excluded until no pair of samples had a kinship coefficient $>0.05$. (xi) missing phenotype information. Following QC, 312 samples with expended/inconsistent C9orf72 status were omitted from further analysis. A total of 5,774 samples (3,955 ALS patients and 1,819 healthy controls) passed all QC and were included in downstream analysis. Per-nucleotide site QC was performed on QCpassing samples only, for Biallelic sites: variants were excluded from analysis based on depth (total DP < 10,000 or $>226,000$ ), missingness $>5 \%$, passing rate in the whole dataset $<70 \%$, sites out of HardyWeinberg equilibrium (HWE; by cohort, controls only, $\mathrm{p}<1 \times 10^{-6}$ ) and sites with extreme differential missingness between cases and control samples (Overall and by cohort, $\mathrm{p}<1 \times 10^{-6}$ ). Non-autosomal chromosomes and multiallelic variants were excluded from analysis.

\section{Selection of regions of interest}

Discontinuous regions of interest approximating in total $\sim 5 \mathrm{Mb}$, include coding sequences and $3^{\prime}$ untranslated regions (3'UTRs) of 295 genes (Supplementary Table 3) encoding for proteins that were: (i) previously reported to be associated with ALS, (ii) RNA-binding proteins including miRNA biogenesis or activity factors [UCSC gene annotation; ${ }^{66}$ ]. In addition to (iii) all 1,750 human pre-miRNA genes [miRBase v20; ${ }^{29}$ ]. In addition, genome-wide analysis of all known human 3 'UTRs (RefSeq ${ }^{36}$ ). Variants in regions of interest were extracted from Project MinE ALS sequencing consortium genomes using vcftools ${ }^{67}$ according to BED file containing genomic coordinates of interest (hg19) \pm 300 bp that ensures covering splice junctions and sequence (Supplementary Table 7). 


\section{Annotation and burden analysis}

After quality control and extraction of regions of interest we performed functional annotation of all variants. Indels were left-aligned and normalized using bcftools and multiallelic sites were removed. For variant annotation we developed a pipeline that calculates the impact of genetic variation in coding regions as well as in $3^{\text {' } U T R ~ a n d ~ m i R N A ~ r e g i o n s, ~ u s i n g ~ A N N O V A R ~}{ }^{68}$. The frequency of the variants in the general population was assessed by screening the 1000 Genomes Project, the Exome Aggregation Consortium (ExAC) and NHLBI Exome Sequencing Project (ESP). For protein coding ORFs, association analysis of deleterious rare variants was performed, i.e., frameshift variants, deviation from canonical splice variant, stop gain/loss variants or a non-synonymous substitution, as predicted by at least three prediction programs (SIFT, Polyphen2 HVAR, LRT, MutationTaster, MutationAssessor, FATHMM, MetaLR) in dbNSFP environment $\left[\mathrm{v} 2.0 ;{ }^{30}\right]$.

Non-coding sequence burden analysis included (i) 3'UTRs, (ii) variants in miRNA recognition elements in 3'UTRs (Supplementary Table 3): Variants that impaired conserved-miRNA binding sites in 3'UTRs (predicted loss of function) were called by TargetScan $\left[\mathrm{v} 7.0 ;{ }^{69}\right]$. Newly created miRNA binding sites in 3'UTRs (predicted gain of function) were called by textual comparison of all possible mutated seeds around a variant to all known miRNA seed sequences in the genome, (iii) all human pre-miRNAs (mirBase $\mathrm{v} 20^{29}$ ) and (iv) miRNAs:target gene networks: mature miRNA sequences (mirBase v20 ${ }^{29}$ ) and cognate targets within the 3'UTRs (Supplementary Table 3).

\section{Mammalian Cell Cultures}

Lymphoblastoid cell lines (LCLs) from the UK MNDA DNA Bank ${ }^{38}$ were originally derived from an individual carrying the suggested IL18RAP protective variant (V3, Chr2:103068718 G>A) and another individual with the canonical IL18RAP 3'UTR (Weizmann IRB: 537-1). LCLs were cultured in RPMI1640 (Gibco, 21875091) with 20\% inactivated fetal bovine serum (FBS, Biological Industries, 04-0011A), $1 \%$ L-glutamine and 1\% penicillin-streptomycin (Biological Industries, $03-0311 \mathrm{~B}$ ) at $37^{\circ} \mathrm{C}, 5 \% \mathrm{CO} 2$. Human Bone Osteosarcoma Epithelial Cells (U2OS), were maintained in Dulbecco’s Modified Eagle Medium (DMEM, Biological Industries, 01-050-1A) supplemented with 10\% FBS, 1\% penicillinstreptomycin at $37^{\circ} \mathrm{C}, 5 \% \mathrm{CO} 2$. 


\section{Cloning}

318 Full IL18RAP coding sequence (CDS) and 3'UTR sequence (2223bp) in pMX vector was purchased from 319 GeneArt (Invitrogen, Supplementary Table 8) and subcloned with V5 epitope into pcDNA3. Different 320 mutants, including: WT ILI8RAP CDS + mutant 3'UTR (V1 or V3), and a dominant negative coding

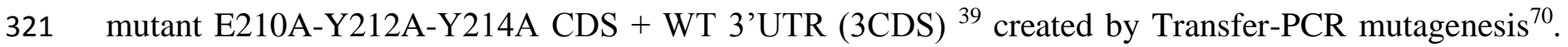
322 Next, WT and mutants full ILI8RAP were subcloned into pUltra vector (a gift from Malcolm Moore, 323 Addgene plasmid \#24130, for which mCherry was replaced with EGFP), downstream of the human 324 Ubiquitin C promoter and EGFP-P2A. Human IL18RAP 3'UTR sequences (422bp) in pMX vector were 325 purchased from GeneArt (Invitrogen, Supplementary Table 8) and subcloned into hRluc reporter in 326 psiCHECK-2 vector (Promega). All cloning procedures were done via restriction free cloning ${ }^{71}$. List of primers used for cloning and Transfer-PCR mutagenesis described in Supplementary Table 9.

\section{Transfection and luciferase assays}

Transfection to U2OS Cells at $1.9 \mathrm{~cm}^{2}$ Corning plates was performed at $70-80 \%$ confluence, $24 \mathrm{~h}$ post plating in antibiotic- free media, using Lipofectamine 2000, 0.5 $\mu \mathrm{L}$ per well (Thermo Fisher Scientific, Cat\# 11668027). Each well was considered as a single replicate. miRNA sensor: U2OS cells were harvested $72 \mathrm{~h}$ post-transfection with human IL18RAP 3'UTR downstream to hRluc reporter (psiCHECK2 vector $500 \mathrm{ng} / 1.9 \mathrm{~cm}^{2}$ plate), for Dual luciferase reporter assay (Promega). NF- $\mathrm{B}$ reporter assay: U2OS cells were induced with recombinant IL-18 $(5 \mathrm{ng} / \mathrm{ml}) 72 \mathrm{~h}$ post-transfection with full coding sequence of ILI8RAP coding region + 3'UTRs (pUltra vector $500 \mathrm{ng} / 1.9 \mathrm{~cm}^{2}$ plate), luc2P/NF- $\mathrm{BB}-\mathrm{RE}$ (pGL4.32 $100 \mathrm{ng}$ ) luciferase and Renilla luciferase (hRluc $10 \mathrm{ng}$ ). $6 \mathrm{~h}$ post later cells were harvested and luminescence quantified.

\section{Cell lysis and Western blot}

339 LCLs were washed in PBSx1, centrifuged at $800 \times g$ for $5 \mathrm{~min}$ at $4^{\circ} \mathrm{C}$, pelleted and lysed in ice-cold RIPA 340 buffer supplemented with cOmplete ${ }^{\mathrm{TM}}$ Protease Inhibitor Cocktail (Roche, 4693116001) and 341 PhosSTOPTM (Roche, 4906837001). The lysates were cleared by centrifugation at 15,000 $\times \mathrm{g}$ for $10 \mathrm{~min}$ 342 at $4^{\circ}$ C. Plasma Membrane Protein Extraction Kit (abcam, ab65400) was used for extraction of membrane343 bound IL18RAP. 
Protein concentrations quantified with Protein Assay Dye Reagent (Bio-Rad, 500-0006), resolved at 50 $\mu \mathrm{g}$ of total protein/well by $10 \%$ polyacrylamide / SDS gel electrophoresis at 100-120 V for 70 min. After gel electrophoresis and transferred to nitrocellulose membrane (Whatmann, 10401383) at 250mA for $70 \mathrm{~min}$. Membranes were stained with Ponceau (Sigma, P7170), blocked for 1 hour at RT with 3\% Bovine albumin fraction V (MPBio 160069) or 5\% milk protein in PBST (PBS containing 0.05\% TWEEN-20) and then incubated with primary antibodies [Rabbit anti IL-18R Beta antibody (Bioss, catalog\# BS-2616R, 1:500), mouse anti GAPDH (Thermo Fisher, catalog\# AM4300, 1:5000), mouse anti p-NFkB p65 antibody (Santa Cruz, catalog\# sc-135769, 1:200), Mouse Anti-beta Actin antibody [AC-15] (abcam, catalog\# ab6276, 1:9,000)] O.N. at $4^{\circ} \mathrm{C}$ with rocking in antibody-Solution [5\% albumin, $0.02 \%$ sodium azide, 5 drops of phenol red in $0.05 \%$ PBST]. Following primary antibody incubation, membranes were washed 3 times for 5 min at RT with $0.05 \%$ PBST then incubated for 1 hour at RT with horseradish peroxidase (HRP)conjugated species-specific secondary antibodies, washed 3 x 5 min in $0.05 \%$ PBST at RT and visualized using EZ-ECL Chemiluminescence (Biological Industries, 20500-120) by ImageQuant ${ }^{\text {TM }}$ LAS 4000 (GE Healthcare Life Sciences). Densitometric analysis performed using ImageJ (NIH).

\section{Induced neuron survival assay}

Survival assay of $H b 9:: \mathrm{RFP}+\mathrm{iMNs}$ was conducted as described previously ${ }^{45}$, with the following modifications: (i) iMNs were infected on day 2 with lentiviruses expressing the full pUltra-IL18RAP constructs, and (ii) longitudinal microscopic tracking was performed every 48 hours, following neurotrophic factor withdrawal and IL-18 treatment $(10 \mathrm{ng} / \mathrm{mL})$, starting on day 15 for 18 additional days. iMN survival assays were performed using three individual replicates / line / condition. iMNs were from two independent donors for each genotype (CTRL/C9-ALS) were combined into one survival trace in the Kaplan-Meier plots for clarity. Ichida lab human lymphocytes from healthy subjects and ALS patients were obtained from the National Institute of Neurological Disorders and Stroke (NINDS) Biorepository at the Coriell Institute for Medical Research and reprogrammed into iPSCs as previously described ${ }^{45}$. The NINDS Biorepository requires informed consent from patients. The experiment involved mouse glial isolation, performed at University of Southern California (USC) and was done in compliance with ethical regulations approved by the USC IACUC committee (Los Angeles, USA).

\section{Statistical analysis}

Statistics performed with Prism Origin (GraphPad). Shapiro-Wilk test was used to assess normality of the data. Pairwise comparisons passing normality test were analyzed with Student's $t$-test, whereas the Mann- 
374 Whitney test was used for pairwise comparison of nonparametric data. Multiple group comparisons were 375 analyzed using ANOVA with post hoc tests. For iMN survival experiments, statistical analysis was 376 performed using a one-sided log-rank test to account for events that did not occur (i.e. iMNs that did not 377 degenerate before the end of the experiment). For each line, the number of iMNs that were analyzed to 378 generate the survival curve is indicted in the figure. Statistical P values $<0.05$ were considered significant. 379 Data are shown as box plots, or as noted in the text. 


\section{Supplementary Materials}

381 Fig. S1. Study design.

382 Fig. S2. Region-based rare-variant association analyses.

383 Fig. S3. 3'UTR-based rare-variant association analysis, using different algorithms.

384 Fig. S4. Evaluation of IL18RAP and IL-18 mRNA expression in motor neurons of patients with ALS.

385 Table S1. Total number of samples before and after quality control procedures, stratified by country.

386 Table S2. Samples Quality Control Procedures.

387 Table S3. Candidate genes list.

388 Table S4. Number of rare qualifying genetics variants identified.

389 Table S5. Identified IL18RAP 3'UTR variants in Project MinE discovery cohort.

390 Table S6. Identified IL18RAP 3'UTR variants in discovery and replication cohorts.

391 Table S7. BED file containing genomic coordinates of regions of interest.

392 Table S8. Synthetic IL18RAP sequences used for cloning into pMX vectors.

393 Table S9. List of primers used for cloning and Transfer-PCR mutagenesis.

394 Data File S1. Detailed description of variants in protein coding sequences of NEK1 and SOD1 and the 395 ILI8RAP 3'UTR, in Project MinE discovery cohort.

396 Data File S2. Source data for IL18RAP western blot studies.

397 Data File S3. Source data for $\mathrm{p}-\mathrm{NF}-\kappa \mathrm{B}$ western blot studies.

398 Project MinE ALS Sequencing Consortium PI List

$399 \quad$ NYGC ALS Consortium PI List 


\section{Acknowledgments:}

401 We gratefully acknowledge the contributions of all participants and the investigators who provided 402 biological samples and data for Project Mine ALS sequencing consortium, the New York Genome Center 403 (NYGC) ALS Consortium, the Genome Aggregation Database (gnomAD) and Trans-Omics for Precision 404 Medicine (TOPMed) of the National Heart, Lung, and Blood Institute (NHLBI, 405 https://www.nhlbiwgs.org/topmed-banner-authorship). Samples used in this research were in part 406 obtained from the UK National DNA Bank for MND Research, funded by the MND Association and the 407 Wellcome Trust. We acknowledge sample management undertaken by Biobanking Solutions funded by 408 the Medical Research Council at the Centre for Integrated Genomic Medical Research, University of 409 Manchester. The authors would like to thank the NINDS Biorepository at Coriell Institute for providing 410 the cell lines used for this study at J.K.I. lab. We thank LSE for language and scientific editing. Hornstein 411 lab is supported by friends of Dr. Sydney Brenner. EH is Head of Nella and Leon Benoziyo Center for 412 Neurological Diseases and incumbent of Ira \& Gail Mondry Professorial chair. Funding: The work is 413 funded by Legacy Heritage Fund, Bruno and Ilse Frick Foundation for Research on ALS, Teva 414 Pharmaceutical Industries Ltd as part of the Israeli National Network of Excellence in Neuroscience (NNE) and Minna-James-Heineman Stiftung through Minerva. The research leading to these results has received funding to E.H. from the European Research Council under the European Union's Seventh Framework Programme (FP7/2007-2013) / ERC grant agreement n 617351. Israel Science Foundation, the ALS-Therapy Alliance, AFM Telethon (20576 to E.H.), Motor Neuron Disease Association (UK), The Thierry Latran Foundation for ALS research, ERA-Net for Research Programmes on Rare Diseases (FP7), A. Alfred Taubman through IsrALS, Yeda-Sela, Yeda-CEO, Israel Ministry of Trade and Industry, Y. Leon Benoziyo Institute for Molecular Medicine, Kekst Family Institute for Medical Genetics, David and Fela Shapell Family Center for Genetic Disorders Research, Crown Human Genome Center, Nathan, Shirley, Philip and Charlene Vener New Scientist Fund, Julius and Ray Charlestein Foundation, Fraida Foundation, Wolfson Family Charitable Trust, Adelis Foundation, MERCK (UK), Maria Halphen, Estates of Fannie Sherr, Lola Asseof, Lilly Fulop. To A.A.C. from Neurodegenerative Disease Research (JPND), Medical Research Council (MR/L501529/1; STRENGTH, MR/R024804/1; BRAIN-MEND), Economic and Social Research Council (ES/L008238/1; ALS-CarE)), MND Association. National Institute for Health Research (NIHR) Biomedical Research Centre at South London and Maudsley NHS Foundation Trust and King's College London. To P.V.D.: Project MinE Belgium was supported by a grant from IWT $\left(n^{\circ}\right.$ 140935), the ALS Liga België, the National Lottery of Belgium and the KU Leuven Opening the 
Future Fund. P.V.D. holds a senior clinical investigatorship of FWO-Vlaanderen and is supported by E. von Behring Chair for Neuromuscular and Neurodegenerative Disorders, the ALS Liga België and the KU Leuven funds "Een Hart voor ALS", "Laeversfonds voor ALS Onderzoek" and the "Valéry Perrier Race against ALS Fund". Several authors of this publication are members of the European Reference Network for Rare Neuromuscular Diseases (ERN-NMD). To P.J.S: from the Medical Research Council, MND Association, NIHR Senior Investigator Award, National Institute for Health Research (NIHR) Sheffield Biomedical Research Centre and NIHR Sheffield Clinical Research Facility. To P.M.A.: Knut and Alice Wallenberg Foundation, the Swedish Brain Foundation, the Swedish Science Council, the UllaCarin Lindquist Foundation. H.P.P. and sequencing activities at NYGC were supported by the ALS Association (ALSA) and The Tow Foundation. C.E. was supported by scholarship from Teva Pharmaceutical Industries Ltd as part of the Israeli National Network of Excellence in Neuroscience (NNE). S.M.K.F. is supported by the ALS Canada Tim E. Noël Postdoctoral Fellowship. R. H. Brown Jr. was funded by ALS Association, ALS Finding a Cure, Angel Fund, ALS-One, Cellucci Fund and NIH grants (R01 NS104022, R01 NS073873 and NS111990-01 to R.H.B.J.). J.K.I. is a New York Stem Cell Foundation-Robertson Investigator. Work at J.K.I. lab was supported by NIH grants R01NS097850, U.S. Department of Defense grant W81XWH-19-PRARP-CSRA, and grants from the Tau Consortium, the New York Stem Cell Foundation, the ALS Association, and the John Douglas French Alzheimer's Foundation. To R.L.McL.: Science Foundation Ireland (17/CDA/4737). To A.N.B.: Suna and Inan Kirac Foundation. To J.E.L.: National Institute of Health/NINDS (R01 NS073873). Author contributions: C.E. led the project; C.E. contributed to research conception, design and interpretations and wrote the manuscript with E.H.; C.E., E.B., T.O., K.R.V.E., S.L.P., M.M., S.M.K.F., N.Y., J.C.-K., K.P.K., R.A.A.V.D.S., W.S., A.A.K., A.I., A.S., A.R.J., E.C., D.R., O.W., R.H.B.J., P.J.S., P.V.D., L.H.V.D.B., H.P.P., E.S., A.A.-C. and J.H.V. collected samples, were involved in the sequence analysis pipeline, phenotyping, variant calling, provided expertise or were involved in the genetic association analysis of rare non-coding variants in human patients with ALS; S.-T.H. and J.K.I. performed iMNs experiments and interpreted data; A.S. and C.E. performed molecular biology studies in LCLs and U2OS cell lines, including reporter assays and protein quantification by western blots; S.W. and D.P.S. helped performing research; E.H. conceived and supervised the study and wrote the manuscript with C.E. All co-authors provided approval of the manuscript. Competing interests: J.K.I. is a co-founder of AcuraStem Incorporated. J.K.I. declares that he is bound by confidentiality agreements that prevent him from disclosing details of his financial interests in this work. E.H. is inventor on pending patent family 
462 PCT/IL2016/050328 entitled "Methods of treating motor neuron diseases". All other authors declare that 463 they have no competing interests. Data availability: Human genetics data is publically available from the 464 sequencing consortia: Project Mine ALS sequencing consortium, the New York Genome Center (NYGC) 465 ALS Consortium, the Genome Aggregation Database (gnomAD) and NHLBI's Trans-Omics for Precision 466 Medicine (TOPMed). All Other data used for this manuscript are available in the manuscript. Code 467 availability: Variant annotation scripts are available at GitHub: https://github.com/TsviyaOlender/Non468 coding-Variants-in-ALS-genes-. 


\section{References}

470

471 1. Cookson, W., Liang, L., Abecasis, G., Moffatt, M. \& Lathrop, M. Mapping complex disease 472 traits with global gene expression. Nature reviews. Genetics 10, 184-194 (2009).

4732 Knight, J.C. Regulatory polymorphisms underlying complex disease traits. Journal of molecular 474 medicine 83, 97-109 (2005).

475 3. Brown, R.H. \& Al-Chalabi, A. Amyotrophic Lateral Sclerosis. The New England journal of 476 medicine 377, 162-172 (2017).

477 4. Taylor, J.P., Brown, R.H., Jr. \& Cleveland, D.W. Decoding ALS: from genes to mechanism. $478 \quad$ Nature 539, 197-206 (2016).

$4795 . \quad$ Renton, A.E., Chio, A. \& Traynor, B.J. State of play in amyotrophic lateral sclerosis genetics. $480 \quad$ Nature neuroscience 17, 17-23 (2014).

481 6. Al-Chalabi, A., van den Berg, L.H. \& Veldink, J. Gene discovery in amyotrophic lateral 482 sclerosis: implications for clinical management. Nat Rev Neurol 13, 96-104 (2017).

483 7. DeJesus-Hernandez, M., et al. Expanded GGGGCC hexanucleotide repeat in noncoding region 484 of C9ORF72 causes chromosome 9p-linked FTD and ALS. Neuron 72, 245-256 (2011).

485 8. Renton, A.E., et al. A hexanucleotide repeat expansion in C9ORF72 is the cause of chromosome 486 9p21-linked ALS-FTD. Neuron 72, 257-268 (2011).

487 9. La Spada, A.R. \& Taylor, J.P. Repeat expansion disease: progress and puzzles in disease 488 pathogenesis. Nature reviews. Genetics 11, 247-258 (2010).

489 10. Haramati, S., et al. miRNA malfunction causes spinal motor neuron disease. Proceedings of the 490 National Academy of Sciences of the United States of America 107, 13111-13116 (2010).

491 11. Emde, A., et al. Dysregulated miRNA biogenesis downstream of cellular stress and ALS-causing 492 mutations: a new mechanism for ALS. The EMBO journal 34, 2633-2651 (2015).

493 12. Eitan, C. \& Hornstein, E. Vulnerability of microRNA biogenesis in FTD-ALS. Brain research 494 (2016).

495 13. Campos-Melo, D., Droppelmann, C.A., He, Z., Volkening, K. \& Strong, M.J. Altered microRNA 496 expression profile in Amyotrophic Lateral Sclerosis: a role in the regulation of NFL mRNA levels.

497 Molecular brain 6, 26 (2013).

498 14. Buratti, E., et al. Nuclear factor TDP-43 can affect selected microRNA levels. The FEBS journal $499 \quad 277,2268-2281(2010)$. 
500 15. Kawahara, Y. \& Mieda-Sato, A. TDP-43 promotes microRNA biogenesis as a component of the 501 Drosha and Dicer complexes. Proceedings of the National Academy of Sciences of the United States of 502 America 109, 3347-3352 (2012).

503 16. Morlando, M., et al. FUS stimulates microRNA biogenesis by facilitating co-transcriptional 504 Drosha recruitment. The EMBO journal 31, 4502-4510 (2012).

505 17. Hoye, M.L., et al. MicroRNA Profiling Reveals Marker of Motor Neuron Disease in ALS 506 Models. J Neurosci 37, 5574-5586 (2017).

507 18. Rotem, N., et al. ALS Along the Axons - Expression of Coding and Noncoding RNA Differs in 508 Axons of ALS models. Sci Rep 7, 44500 (2017).

509 19. Butovsky, O., et al. Modulating inflammatory monocytes with a unique microRNA gene 510 signature ameliorates murine ALS. J Clin Invest 122, 3063-3087 (2012).

511 20. Figueroa-Romero, C., et al. Expression of microRNAs in human post-mortem amyotrophic 512 lateral sclerosis spinal cords provides insight into disease mechanisms. Mol Cell Neurosci 71, 34-45 513 (2016).

514 21. Williams, A.H., et al. MicroRNA-206 delays ALS progression and promotes regeneration of 515 neuromuscular synapses in mice. Science 326, 1549-1554 (2009).

516 22. Bartel, D.P. MicroRNAs: target recognition and regulatory functions. Cell 136, $215-233$ (2009).

517 23. Mayr, C. Regulation by 3'-Untranslated Regions. Annual review of genetics 51, 171-194 (2017).

518 24. Povysil, G., et al. Rare-variant collapsing analyses for complex traits: guidelines and 519 applications. Nature reviews. Genetics 20, 747-759 (2019).

520 25. An, J.Y., et al. Genome-wide de novo risk score implicates promoter variation in autism 521 spectrum disorder. Science 362 (2018).

522 26. Lee, S., Abecasis, G.R., Boehnke, M. \& Lin, X. Rare-variant association analysis: study designs 523 and statistical tests. American journal of human genetics 95, 5-23 (2014).

524 27. Project MinE Consortium, Van Rheenen, W. \& et al. Project MinE: study design and pilot 525 analyses of a large-scale whole-genome sequencing study in amyotrophic lateral sclerosis. (2017).

526 28. Dunckley, T., et al. Whole-genome analysis of sporadic amyotrophic lateral sclerosis. The New 527 England journal of medicine 357, 775-788 (2007).

528 29. Griffiths-Jones, S., Grocock, R.J., van Dongen, S., Bateman, A. \& Enright, A.J. miRBase: 529 microRNA sequences, targets and gene nomenclature. Nucleic acids research 34, D140-144 (2006). 
530 30. Liu, X., Jian, X. \& Boerwinkle, E. dbNSFP v2.0: a database of human non-synonymous SNVs 531 and their functional predictions and annotations. Human mutation 34, E2393-2402 (2013).

532 31. Lee, S., et al. Optimal unified approach for rare-variant association testing with application to 533 small-sample case-control whole-exome sequencing studies. American journal of human genetics $\mathbf{9 1}$, $534 \quad 224-237(2012)$.

535 32. Kenna, K.P., et al. NEK1 variants confer susceptibility to amyotrophic lateral sclerosis. Nature 536 genetics 48, 1037-1042 (2016).

537 33. Rosen, D.R., et al. Mutations in $\mathrm{Cu} / \mathrm{Zn}$ superoxide dismutase gene are associated with familial 538 amyotrophic lateral sclerosis. Nature 362, 59-62 (1993).

539 34. Chio, A., et al. Prevalence of SOD1 mutations in the Italian ALS population. Neurology 70, 533540537 (2008).

541 35. van der Spek, R.A.A., et al. The project MinE databrowser: bringing large-scale whole-genome 542 sequencing in ALS to researchers and the public. Amyotrophic lateral sclerosis \& frontotemporal 543 degeneration 20, 432-440 (2019).

544 36. O'Leary, N.A., et al. Reference sequence (RefSeq) database at NCBI: current status, taxonomic 545 expansion, and functional annotation. Nucleic acids research 44, D733-745 (2016).

546 37. Alboni, S., Cervia, D., Sugama, S. \& Conti, B. Interleukin 18 in the CNS. Journal of 547 neuroinflammation 7, 9 (2010).

548 38. Smith, L., et al. Establishing the UK DNA Bank for motor neuron disease (MND). BMC Genet 549 16, 84-84 (2015).

550 39. Tsutsumi, N., et al. The structural basis for receptor recognition of human interleukin-18. Nature 551 communications 5, 1-13 (2014).

552 40. Kadhim, H., Deltenre, P., Martin, J.J. \& Sebire, G. In-situ expression of Interleukin-18 and 553 associated mediators in the human brain of sALS patients: Hypothesis for a role for immune554 inflammatory mechanisms. Medical hypotheses 86, 14-17 (2016).

555 41. Johann, S., et al. NLRP3 inflammasome is expressed by astrocytes in the SOD1 mouse model of 556 ALS and in human sporadic ALS patients. Glia 63, 2260-2273 (2015).

557 42. Italiani, P., et al. Evaluating the levels of interleukin-1 family cytokines in sporadic amyotrophic 558 lateral sclerosis. Journal of neuroinflammation 11, 94 (2014).

559 43. Krach, F., et al. Transcriptome-pathology correlation identifies interplay between TDP-43 and 560 the expression of its kinase CK1E in sporadic ALS. Acta neuropathologica 136, 405-423 (2018). 
44. Thompson, L. iMN (Exp 2)—ALS. SMA and control (unaffected) iMN cell lines differentiated from iPS cell lines using a long differentiation protocol-RNA-seq., LINCS (collection) (2017).
45. Shi, Y., et al. Haploinsufficiency leads to neurodegeneration in C9ORF72 ALS/FTD human induced motor neurons. Nature medicine 24, 313 (2018).

46. Ayers, K.L., et al. A loss of function variant in CASP7 protects against Alzheimer's disease in homozygous APOE epsilon4 allele carriers. BMC genomics 17 Suppl 2, 445 (2016).

47. Benitez, B.A., et al. Missense variant in TREML2 protects against Alzheimer's disease. Neurobiology of aging 35, 1510 e1519-1526 (2014).

48. Jonsson, T., et al. A mutation in APP protects against Alzheimer's disease and age-related cognitive decline. Nature 488, 96-99 (2012).

49. Sims, R., et al. Rare coding variants in PLCG2, ABI3, and TREM2 implicate microglialmediated innate immunity in Alzheimer's disease. Nature genetics (2017). 50. Landers, J.E., et al. Reduced expression of the Kinesin-Associated Protein 3 (KIFAP3) gene increases survival in sporadic amyotrophic lateral sclerosis. Proceedings of the National Academy of Sciences of the United States of America 106, 9004-9009 (2009).

51. Farhan, S.M.K., et al. Exome sequencing in amyotrophic lateral sclerosis implicates a novel gene, DNAJC7, encoding a heat-shock protein. Nature neuroscience 22, 1966-1974 (2019).

52. Lambrechts, D., et al. VEGF is a modifier of amyotrophic lateral sclerosis in mice and humans and protects motoneurons against ischemic death. Nature genetics 34, 383-394 (2003).

53. Beers, D.R. \& Appel, S.H. Immune dysregulation in amyotrophic lateral sclerosis: Mechanisms and emerging therapies. The Lancet Neurology 18, 211-220 (2019).

54. Engelhardt, J.I. \& Appel, S.H. IgG reactivity in the spinal cord and motor cortex in amyotrophic lateral sclerosis. Archives of neurology 47, 1210-1216 (1990).

55. Engelhardt, J.I., Tajti, J. \& Appel, S.H. Lymphocytic infiltrates in the spinal cord in amyotrophic lateral sclerosis. Archives of neurology 50, 30-36 (1993).

56. Henkel, J.S., et al. Presence of dendritic cells, MCP-1, and activated microglia/macrophages in amyotrophic lateral sclerosis spinal cord tissue. Annals of neurology 55, 221-235 (2004).

57. Philips, T. \& Robberecht, W. Neuroinflammation in amyotrophic lateral sclerosis: role of glial activation in motor neuron disease. The Lancet. Neurology 10, 253-263 (2011). 
58. Zhernakova, A., et al. Genetic analysis of innate immunity in Crohn's disease and ulcerative colitis identifies two susceptibility loci harboring CARD9 and IL18RAP. American journal of human genetics 82, 1202-1210 (2008).

59. Hirota, T., et al. Genome-wide association study identifies eight new susceptibility loci for atopic dermatitis in the Japanese population. Nature genetics 44, 1222-1226 (2012).

60. Liu, H., et al. Identification of IL18RAP/IL18R1 and IL12B as leprosy risk genes demonstrates shared pathogenesis between inflammation and infectious diseases. American journal of human genetics 91, 935-941 (2012).

61. Hunt, K.A., et al. Newly identified genetic risk variants for celiac disease related to the immune response. Nature genetics 40, 395-402 (2008).

62. Smyth, D.J., et al. Shared and distinct genetic variants in type 1 diabetes and celiac disease. The New England journal of medicine 359, 2767-2777 (2008).

63. Leal-Lasarte, M.M., Franco, J.M., Labrador-Garrido, A., Pozo, D. \& Roodveldt, C. Extracellular TDP-43 aggregates target MAPK/MAK/MRK overlapping kinase (MOK) and trigger caspase-3/IL-18 signaling in microglia. FASEB journal : official publication of the Federation of American Societies for Experimental Biology 31, 2797-2816 (2017).

64. Zhan, X., Hu, Y., Li, B., Abecasis, G.R. \& Liu, D.J. RVTESTS: an efficient and comprehensive tool for rare variant association analysis using sequence data. Bioinformatics 32, 1423-1426 (2016).

65. Raczy, C., et al. Isaac: ultra-fast whole-genome secondary analysis on Illumina sequencing platforms. Bioinformatics 29, 2041-2043 (2013).

66. Tyner, C., et al. The UCSC Genome Browser database: 2017 update. Nucleic acids research 45, D626-D634 (2017).

67. Danecek, P., et al. The variant call format and VCFtools. Bioinformatics 27, 2156-2158 (2011).

68. Wang, K., Li, M. \& Hakonarson, H. ANNOVAR: functional annotation of genetic variants from high-throughput sequencing data. Nucleic acids research 38, e164 (2010).

69. Agarwal, V., Bell, G.W., Nam, J.W. \& Bartel, D.P. Predicting effective microRNA target sites in mammalian mRNAs. eLife 4 (2015).

70. Erijman, A., Dantes, A., Bernheim, R., Shifman, J.M. \& Peleg, Y. Transfer-PCR (TPCR): a highway for DNA cloning and protein engineering. Journal of structural biology 175, 171-177 (2011). 71. Peleg, Y. \& Unger, T. Application of the Restriction-Free (RF) cloning for multicomponents assembly. Methods in molecular biology 1116, 73-87 (2014). 
bioRxiv preprint doi: https://doi.org/10.1101/2021.06.03.446863; this version posted June 5, 2021. The copyright holder for this preprint (which was not certified by peer review) is the author/funder. All rights reserved. No reuse allowed without permission.

\section{FIGURES}


(i) Rare deleterious variants in protein coding ORFs

\section{Exon1}

Exon2

Exon3

(ii) Rare variants in miRNA recognition elements in 3'UTRs Gene 1 $\operatorname{miR} X$ $\operatorname{miR} Y$ miR Z

\section{3'UTR}

(iii) Rare variants in pre-miRNA genes

\section{pre-miRNA X}

(iv) Rare variants in mature miRNA:target genes network

\section{Mature miRNA X}

Gene 1

$$
\operatorname{miR} X
$$

Gene 1

Gene 1

\section{$\operatorname{miR} X$}

7
6

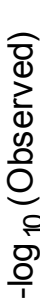

7

$-1$

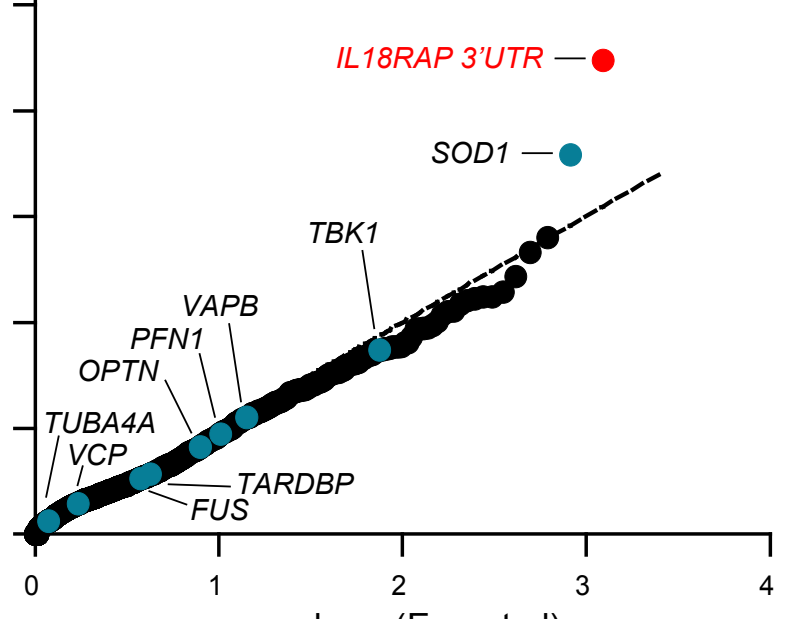
$-\log _{10}($ Expected $)$ 
622 Fig. 1. Region-based rare-variant association analysis. (A) Diagram for region-based rare-variant 623 association studies. Collapsed region-based association analysis was performed on rare (MAF $\leq 0.01)$ 624 qualifying variants in: (i) 295 candidate protein-coding genes (Supplementary Table 3) encoding for ALS625 relevant proteins or proteins associated with miRNA biogenesis/activity. Variants were included if 626 predicted to cause frameshifting, alternative splicing, an abnormal stop codon, or a deleterious non627 synonymous amino acid substitution, in $\geq 3$ of 7 independent dbNSFP prediction algorithms; (ii) variants 628 abrogating or gaining miRNA recognition elements in 3'UTRs of the 295 genes (Supplementary Table 3); 629 (iii) all known pre-miRNA genes in the human genome; and (iv) predicted networks, comprised of 630 aggregated variants detected in a specific mature miRNA sequence and its cognate down-stream 3'UTR 631 targets. (B) QQ plot of obtained and expected P-values for the burden of rare variants (log scale) gained 632 by collapsed region-based association analysis of all genomic regions described in (A). Data were obtained 633 from 3,955 ALS cases and 1,819 controls (Project MinE). Features positioned on the diagonal line 634 represent results obtained under the null hypothesis. Open-reading frames of 10 known ALS genes (blue). 635 IL18RAP 3'UTR miRNA recognition elements (red). Genomic inflation $\lambda=1.21$. 


\begin{tabular}{ll|cccccc}
\hline \multicolumn{1}{c|}{ Gene } & \multicolumn{1}{c|}{ Region } & $\begin{array}{c}\text { ALS } \\
\text { (3955) }\end{array}$ & $\begin{array}{c}\text { Control } \\
(\mathbf{1 8 1 9 )}\end{array}$ & OR & OR 95\% Cl & P & P corrected \\
\hline NEK1 & Coding & 127 & 19 & 3.14 & $1.93-5.11$ & $7.04 \times 10^{-7}$ & $2.13 \times 10^{-4}$ \\
SOD1 & Coding & 36 & 0 & 33.89 & $2.08-552.47$ & $2.61 \times 10^{-4}$ & $3.76 \times 10^{-2}$ \\
IL18RAP & $\begin{array}{l}\text { miRNA binding sites } \\
\text { in 3'UTR }\end{array}$ & 4 & 9 & 0.20 & $0.06-0.66$ & $3.34 \times 10^{-5}$ & $9.31 \times 10^{-3}$ \\
IL18RAP & 3'UTR & 6 & 12 & 0.23 & $0.09-0.61$ & $1.88 \times 10^{-5}$ & $5.62 \times 10^{-3}$ \\
\hline
\end{tabular}

B

\begin{tabular}{lcccc}
\hline \multicolumn{1}{c}{ Cohort } & Cases & Control & OR & OR 95\% Cl \\
\hline Ireland & $0 / 239$ & $1 / 136$ & 0.19 & $0.008-4.662$ \\
Netherlands & $0 / 1633$ & $4 / 1004$ & 0.07 & $0.004-1.265$ \\
Turkey & $0 / 142$ & $1 / 67$ & 0.16 & $0.006-3.870$ \\
UnitedKingdom & $2 / 1043$ & $2 / 272$ & 0.26 & $0.036-1.850$ \\
USA & $2 / 398$ & $1 / 68$ & 0.34 & $0.030-3.784$ \\
Belgium & $0 / 295$ & $0 / 172$ & - & - \\
Spain & $0 / 205$ & $0 / 100$ & - & - \\
\hline Total & $4 / 3955$ & $9 / 1819$ & 0.20 & $0.063-0.662$ \\
\hline
\end{tabular}

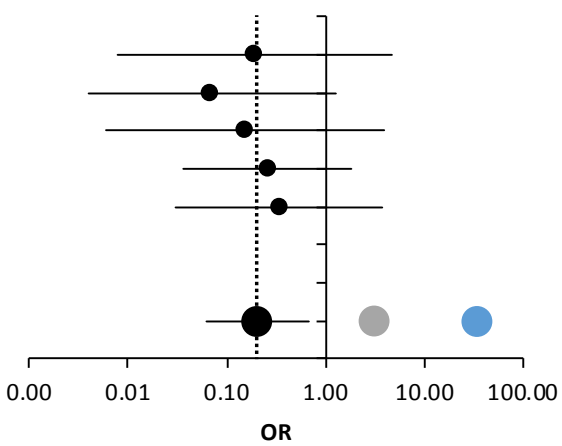

C

\begin{tabular}{|c|c|c|c|c|c|c|}
\hline \multirow{2}{*}{ Cohort } & \multirow{2}{*}{ Cases } & \multirow{2}{*}{ Control } & \multirow{2}{*}{ OR } & \multirow{2}{*}{$95 \% \mathrm{Cl}$} & \multicolumn{2}{|c|}{$\mathbf{P}$} \\
\hline & & & & & $\chi 2$ & SKAT-O \\
\hline $\begin{array}{l}\text { Discovery: } \\
\text { Project MinE }\end{array}$ & $6 / 3955$ & $12 / 1819$ & 0.23 & $0.086-0.611$ & $3.00 \times 10^{-3}$ & $1.88 \times 10^{-5}$ \\
\hline $\begin{array}{l}\text { Replication: } \\
\text { NYGC, TOPMed \& gnomAD }\end{array}$ & $8 / 2184$ & $786 / 68584$ & 0.32 & $0.158-0.637$ & $9.58 \times 10^{-4}$ & - \\
\hline $\begin{array}{l}\text { Joint analysis: } \\
\text { Discovery \& replication }\end{array}$ & $14 / 6139$ & 798/70403 & 0.20 & $0.118-0.338$ & $<1.00 \times 10^{-5}$ & - \\
\hline
\end{tabular}

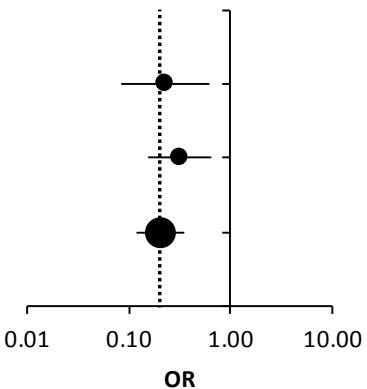


636 Fig 2. Odds of ALS is reduced with rare variants in the ILI8RAP 3'UTR. (A) Odds ratio (OR) 637 estimates with 95\% confidence intervals (CI) for NEK1 (coding), SOD1 (coding), predicted miRNA 638 recognition elements in the IL18RAP 3'UTR, and for all variants identified in the IL18RAP 3'UTR. P 639 values corrected for false discovery rate (FDR). (B) Stratification of data pertaining to miRNA recognition 640 elements in the IL18RAP 3'UTR in seven geographically-based sALS sub-cohorts and forest plot (Log 641 scale). NEK1 (grey) and SOD1 (blue) signals are from combined data of all cohorts. Vertical dotted line 642 denotes $\mathrm{OR}=0.2$. (C) OR with 95\% CI and forest plot (Log scale) across discovery and replication cohorts 643 and joint analysis thereof. Vertical dotted line denotes OR=0.2. P-values, calculated with SKAT-O or Chi644 squared test with Yate's correction. 
Human IL18RAP

ENST00000264260 Chr2:103035149-103069025 [GRCh37/hg19]

3'UTR length: 384 Chr2:103068641-103069025 [GRCh37/hg19]

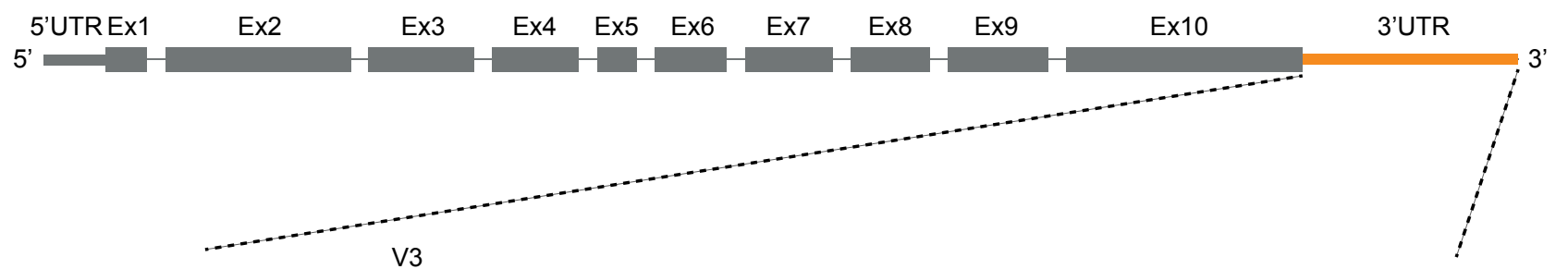

miR-329-3p/362-3P

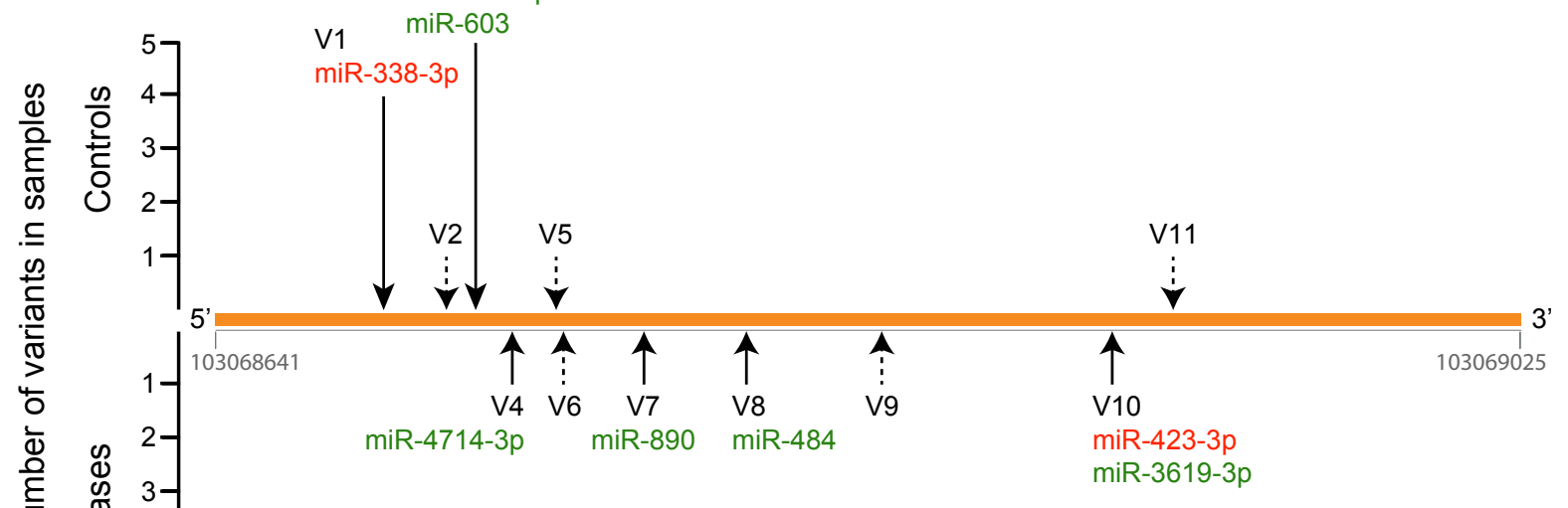

B

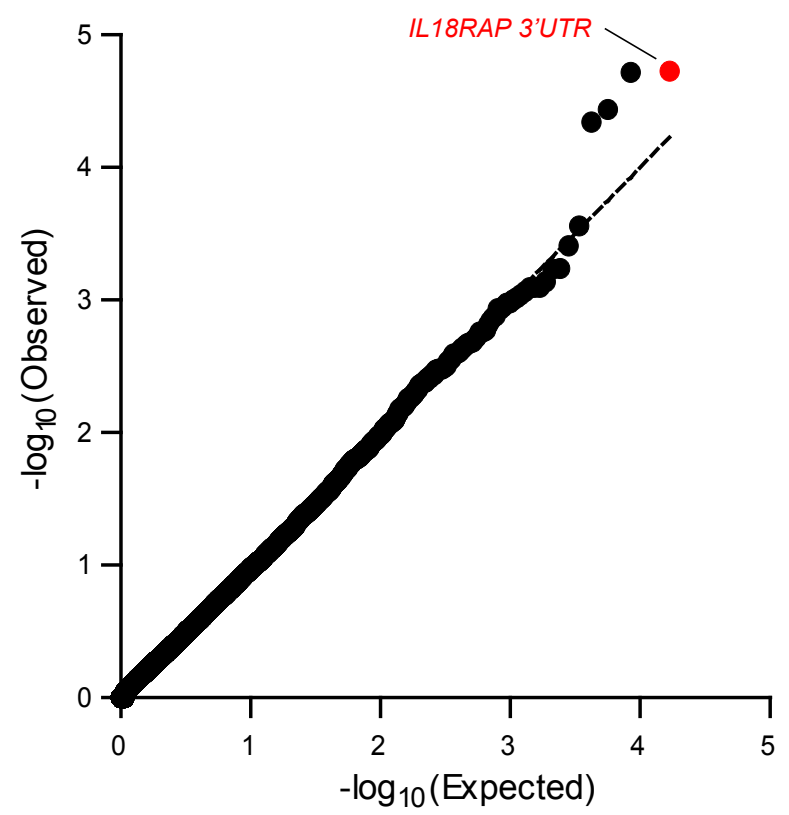


645 Fig. 3. Rare variants in the ILI8RAP 3'UTR. (A) Schematic of the ILI8RAP transcript and 3'UTR (5' 646 to $3^{\prime}$ ) showing the number of control (upper) or ALS (lower) samples, in which miRNA recognition 647 element variants (black arrow) or other variants (dashed arrow) were identified. Potentially lost (red) or 648 created (green) miRNA recognition elements are marked (Supplementary Table 6). (B) QQ plot of 649 obtained and expected P-values for the burden of rare variants (log scale) gained by collapsed region650 based association analysis for all known human 3'UTRs (RefSeq), in Project MinE cohort (3,955 ALS 651 cases and 1,819 non-ALS controls). Variants are not restricted to miRNA recognition elements. Features 652 positioned on the diagonal line represent results obtained under the null hypothesis. The IL18RAP 3'UTR 653 (red) is the most significant 3'UTR associated with ALS. Genomic inflation $\lambda=0.97$. 

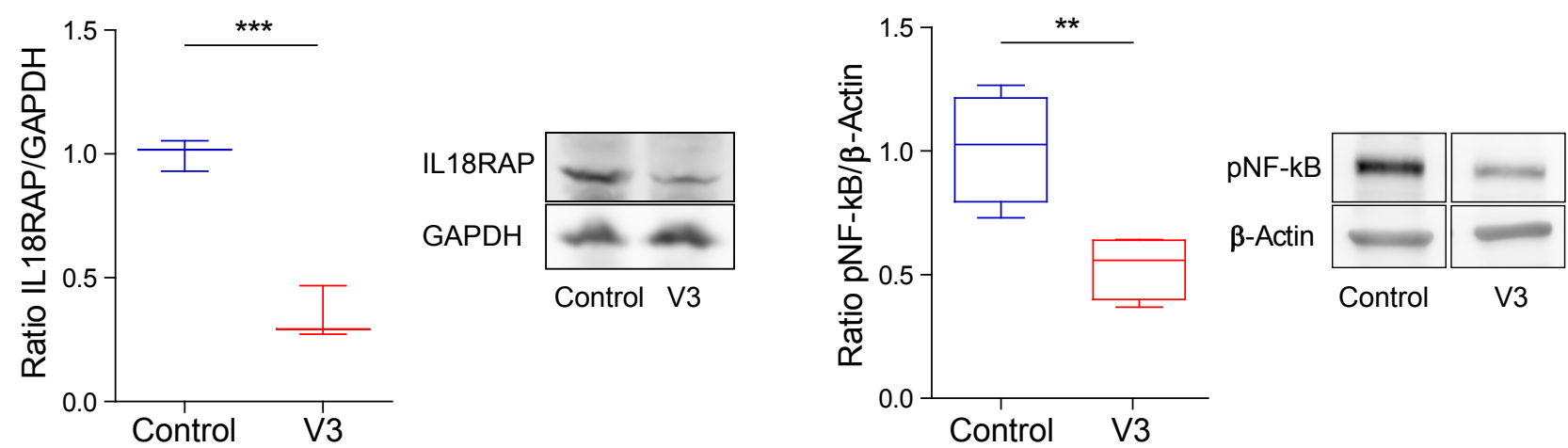

\section{Biosensor for 3'UTR variant activity}
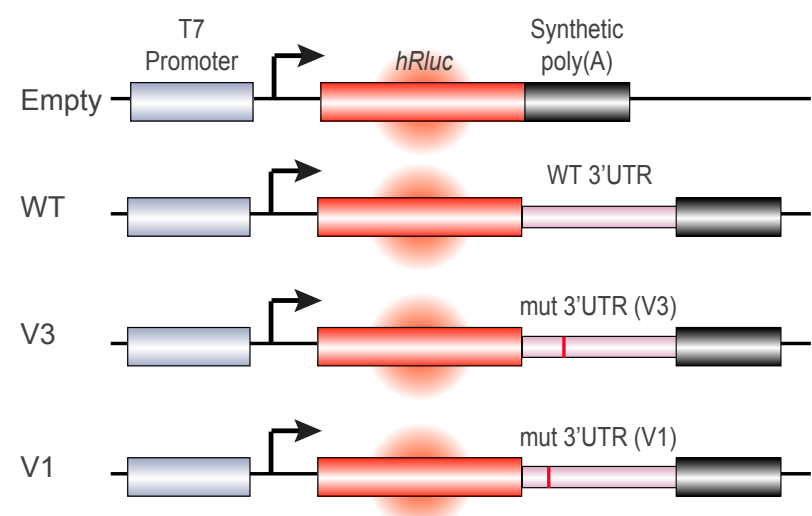

E Biosensor for NF-kB pathway activity
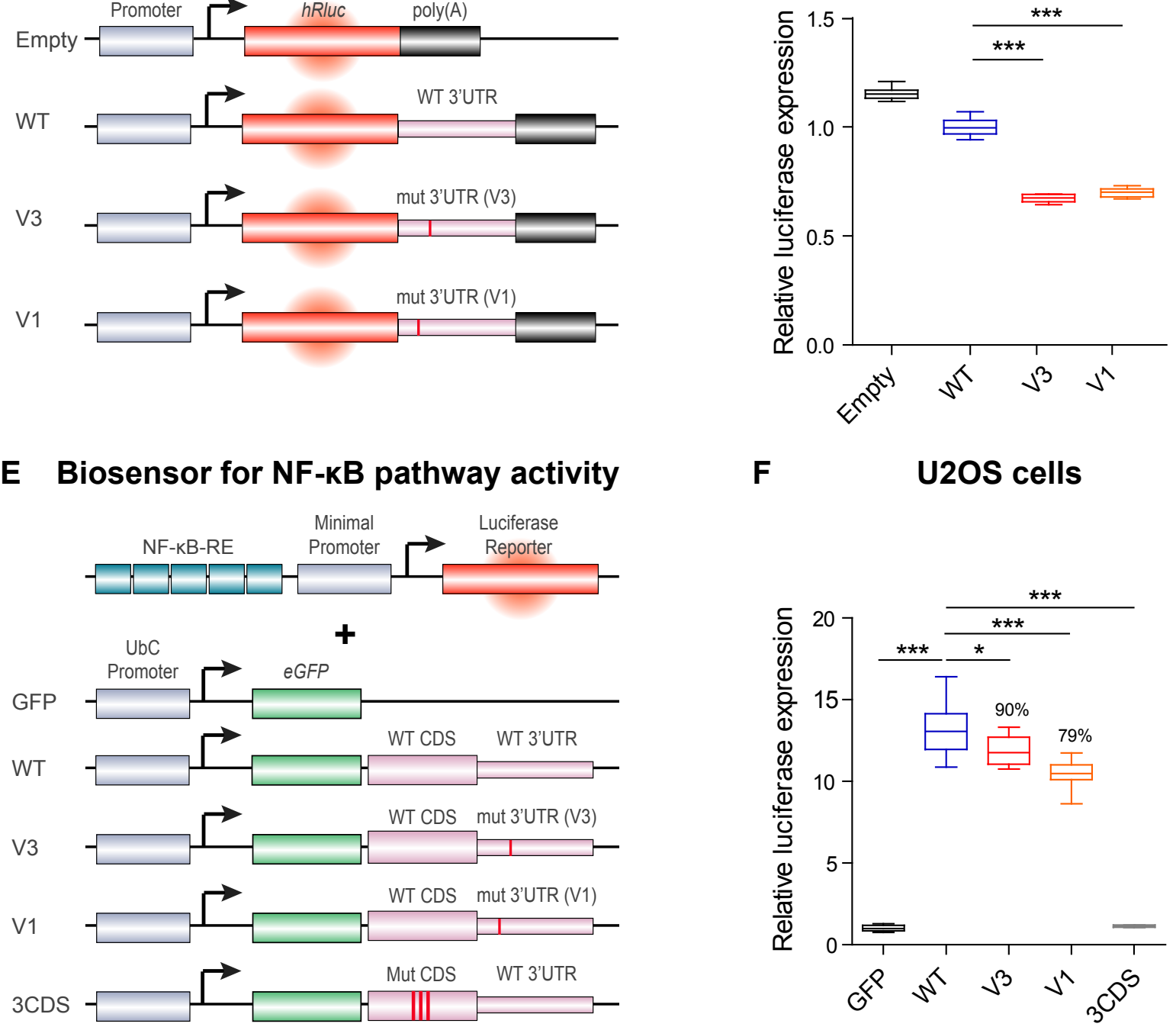
654 Fig 4. ILI8RAP 3'UTR variant attenuates IL-18 / NF-kB signaling. Quantification of protein 655 expression for IL18RAP $(n=3)(A)$ or $p-N F-\kappa B$ protein $(n=4)(B)$ by Western blots of extracts from a 656 lymphoblastoid cell line harboring an endogenous IL18RAP variant (V3, Chr2:103068718 G>A) relative 657 to IL18RAP protein in a line with the canonical ILI8RAP 3'UTR. Loading normalization with anti 658 GAPDH or anti Beta-Actin. Two-sided student $t$-test. Diagram (C) and quantification (D) of hRluc 659 Il18RAP 3'UTR reporter assays, in human U2OS cell line (Empty, WT, V3, V1; n=6). One-way ANOVA 660 followed by Dunnett's multiple comparison test. Diagram (E) and quantification (F) of NF- $\kappa B$ reporter 661 assay in human U2OS cell line (GFP, WT, V3, V1, n=9; 3CDS, n=4). One-way ANOVA followed by 662 Dunnett's multiple comparison test was performed on square root-transformed data. Box plots depicting 663 median, upper and lower quartiles, and extreme points. $* \mathrm{P}<0.05 ; * * \mathrm{P}<0.01 ; * * * \mathrm{P}<0.001$. Experiment 664 repeated independently three times with similar results. 


\section{A C9ORF72 patients iMNs}
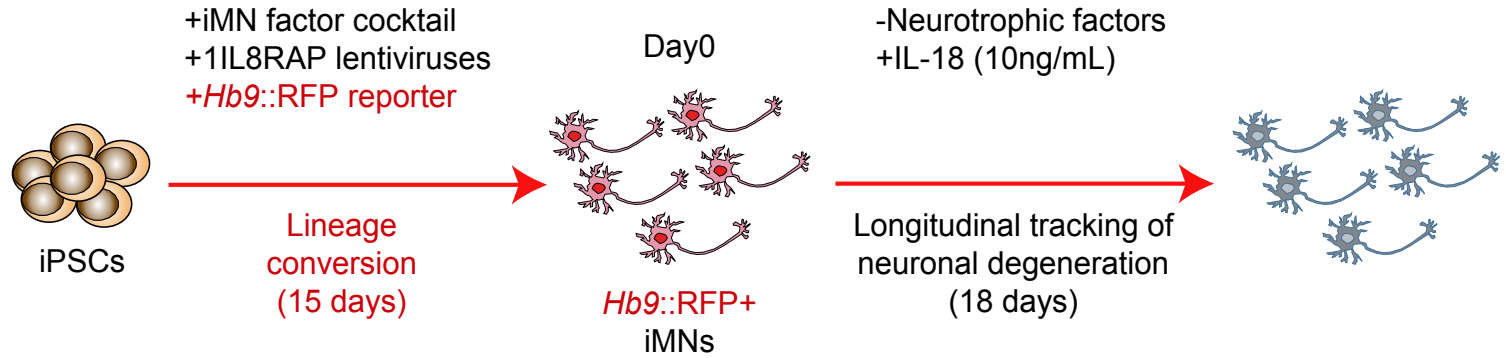

B

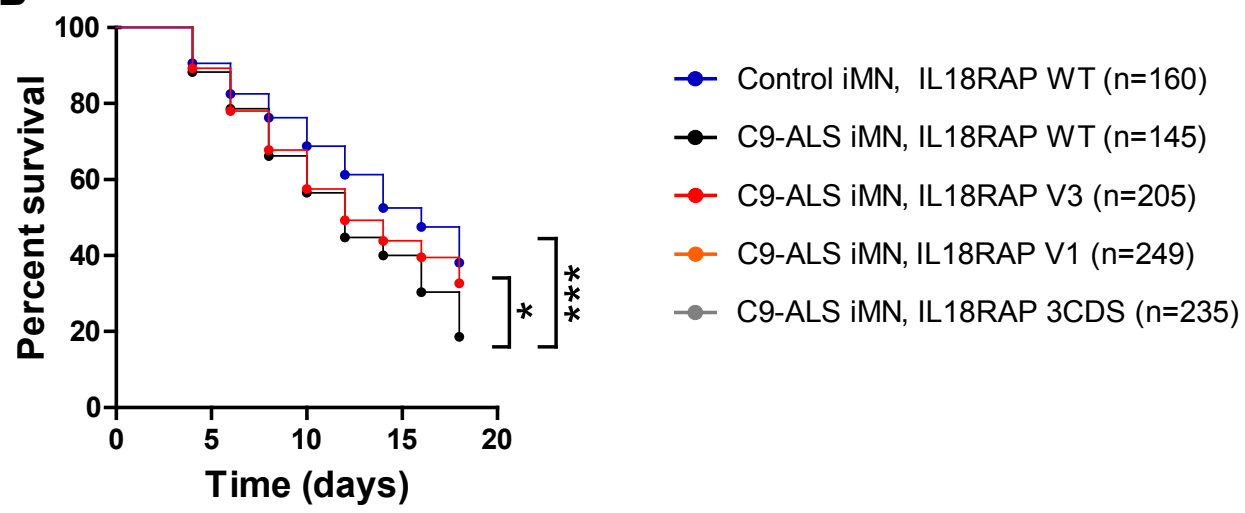

C

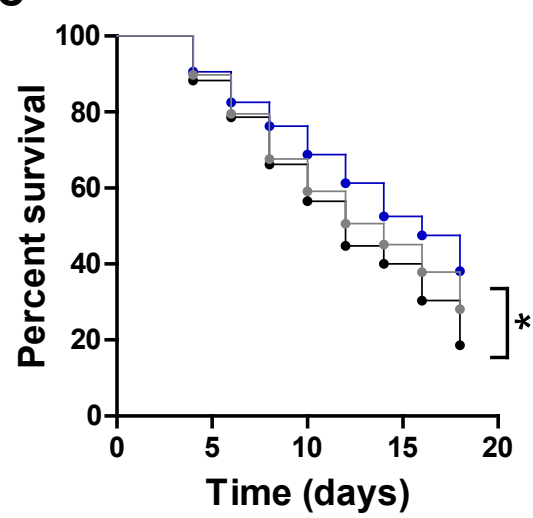

$\mathbf{F}$

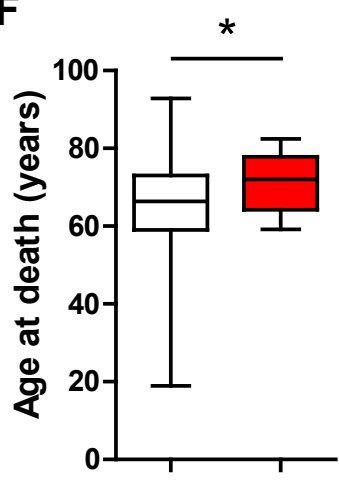

ALS ALS with

Cohort IL18RAP variants
D

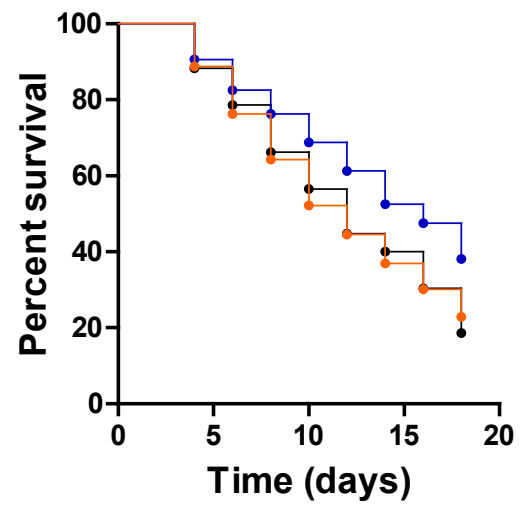

G

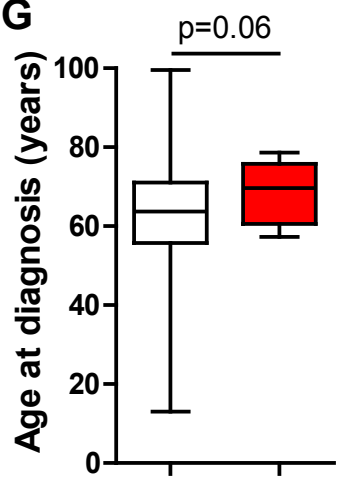

ALS ALS with Cohort IL18RAP
E

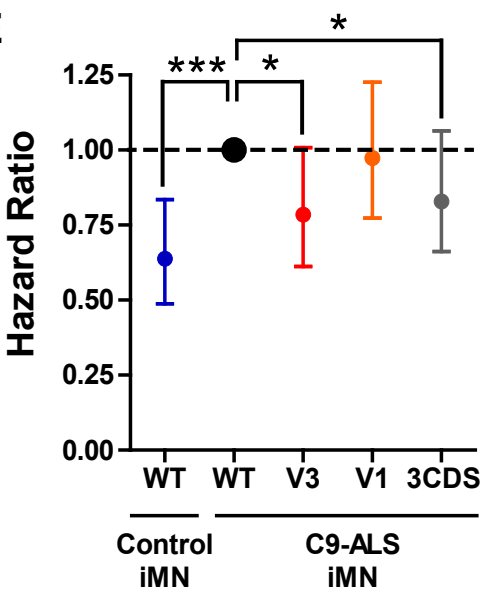


665 Fig 5. ILI8RAP 3'UTR variant ameliorates disease in C9orf72 patient iMNs and in patients with

666 ALS. Experimental setup: Time-lapse survival tracking microscopy of $H b 9:: \mathrm{RFP}+\mathrm{iMNs}$, transduced 667 with IL18RAP lentiviruses, after neurotrophic factors withdrawal and introduction of IL-18 cytokine 668 (A). Kaplan-Meier survival plots for control (CTRL) and C9orf72 patient (C9-ALS) iMNs, with wild669 type (WT) IL18RAP, IL18RAP harboring variants in the 3'UTR (V3, V1) or an IL18RAP dominant 670 negative coding mutant (E210A-Y212A-Y214A) 3CDS. Traces of iMNs from 2 donors per genotype 671 (control/ C9-ALS lines), quantified from 3 independent iMN differentiation experiments per line. 672 Number of iMNs quantified per treatment denoted. One-sided log-rank test for the entire survival time 673 course (B-D) and corresponding hazard ratio of cellular death, relative to C9-ALS iMNs with wild-type 674 IL18RAP 3'UTR (E). Association of age of death (9 patients with protective 3'UTR variants /4263 675 patients with available phenotypic data in Project MinE and NYGC cohorts (F) or diagnosis (8/4216 676 patients) (G). IL18RAP variant is associated with delayed age of death $(+6.1$ years, * $\mathrm{P}<0.05)$ and age of 677 diagnosis (+6.2 years, subthreshold significance of $\mathrm{P}=0.06)$, relative to the mean age of all Project 678 MinE and NYGC ALS patients. Box plots depicting median, upper and lower quartiles, and extreme 679 points, one-sided Mann-Whitney test. * $\mathrm{P}<0.05 ; * * * \mathrm{P}<0.001$. 
WGS sequencing, variant calling and QC

$$
\downarrow
$$

\section{Capture regions of interest from WGS}

$\downarrow$

Bioinformatics assay

and functional annotation

$\downarrow$

Region-based rare-variant

association analysis

Genetic and biological validation

ALS Control

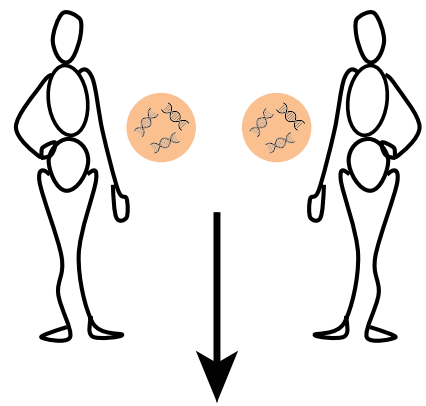

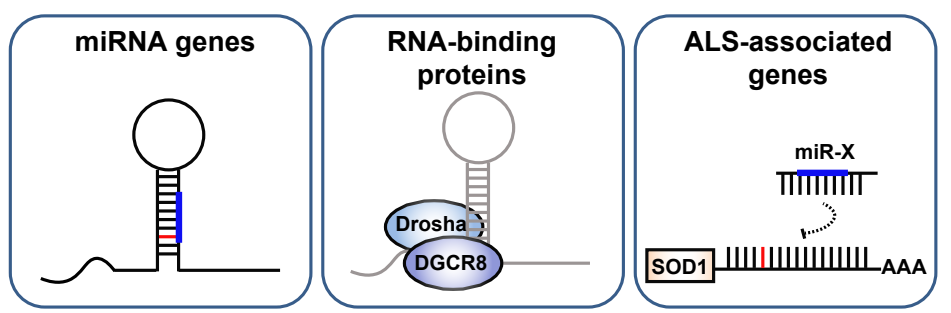

\section{Supplementary Fig.1 - Eitan et al. (Hornstein)}


680 Supplementary Fig. 1. Study design. (A) Flow chart of approach for discovery of region-based rare681 variants in non-coding genomic regions via association studies and (B) diagram depicting regions of 682 interest comprising of 1,750 human pre-miRNA genes, 295 open reading frames encoding for proteins of 683 interest and 295 3'UTRs. 
684 Supplementary Fig. 2. Region-based rare-variant association analyses. (A-D) QQ plot of obtained 685 and expected $P$-values for the burden of rare-variants (log scale) gained by collapsed region-based 686 association analysis of different genomic regions, comprised of (A) 295 candidate protein-coding listed 687 in Supplementary Table 3. These ORFs encode for ALS-relevant proteins or proteins that are associated 688 with miRNA biogenesis or activity. Variants were depicted if predicted to cause frameshifting, alternative 689 splicing, abnormal stop codon or a deleterious non-synonymous amino acid substitution, in $\geq 3$ of 7 690 independent dbNSFP prediction algorithms (genomic inflation $\lambda=0.97$ ), (B) All known pre-miRNA genes 691 in the human genome (genomic inflation $\lambda=1.30$ ), (C) predicted networks, comprised of aggregated 692 variants detected on a specific mature miRNA sequence and its cognate downstream 3'UTR targets 693 (genomic inflation $\lambda=1.17$ ), and (D) variants abrogating or gaining miRNA recognition elements in 694 3'UTRs of same 295 genes listed in Supplementary Table 3 (genomic inflation $\lambda=1.03$ ). Data was 695 obtained from 3,955 ALS cases and 1,819 controls (Project MinE). Features positioned on the diagonal 696 line represent results obtained under the null hypothesis. Open-reading frames of 10 known ALS genes 697 (blue). ILI8RAP 3'UTR miRNA recognition elements (red). 

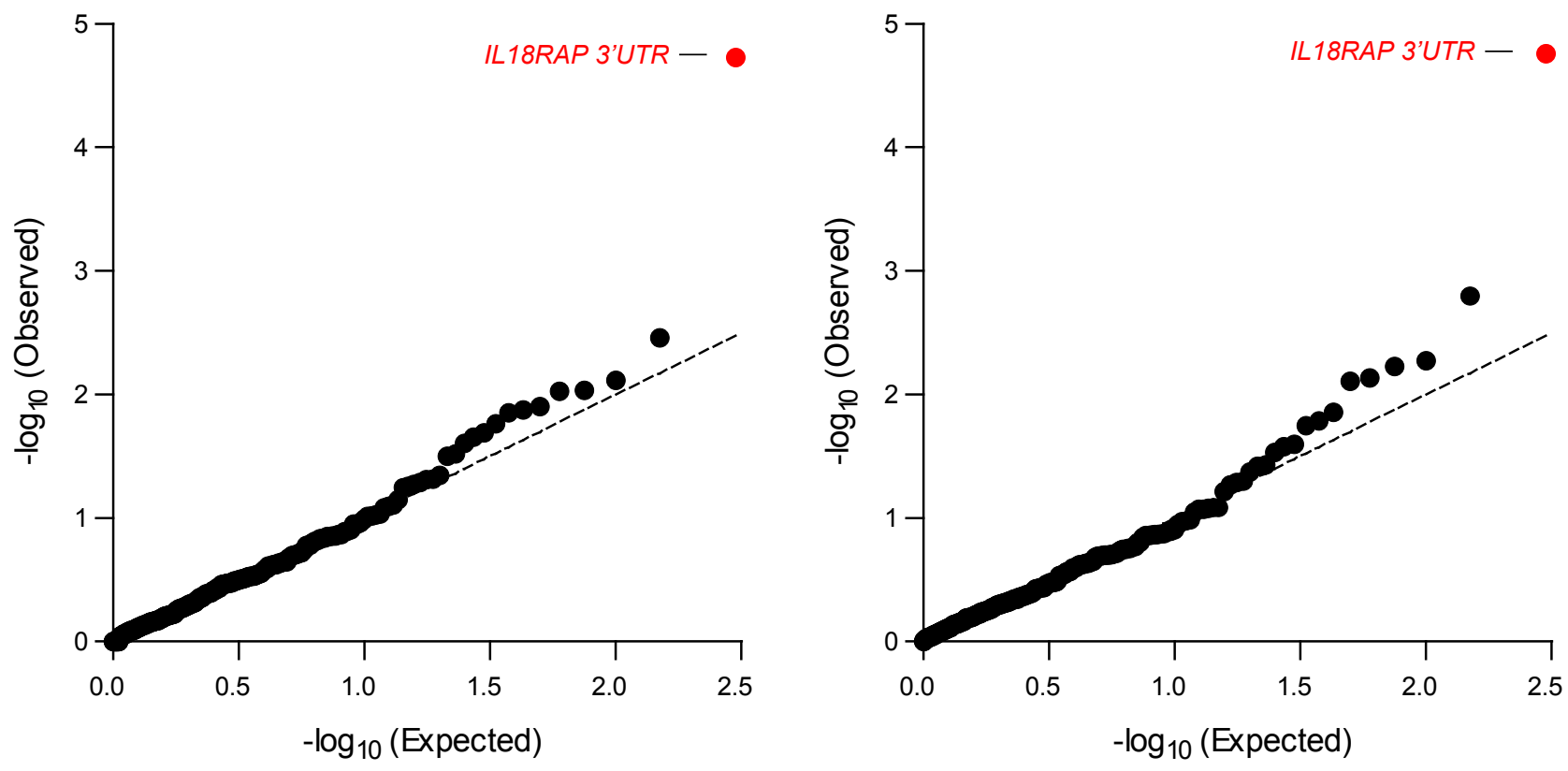

C

CMC

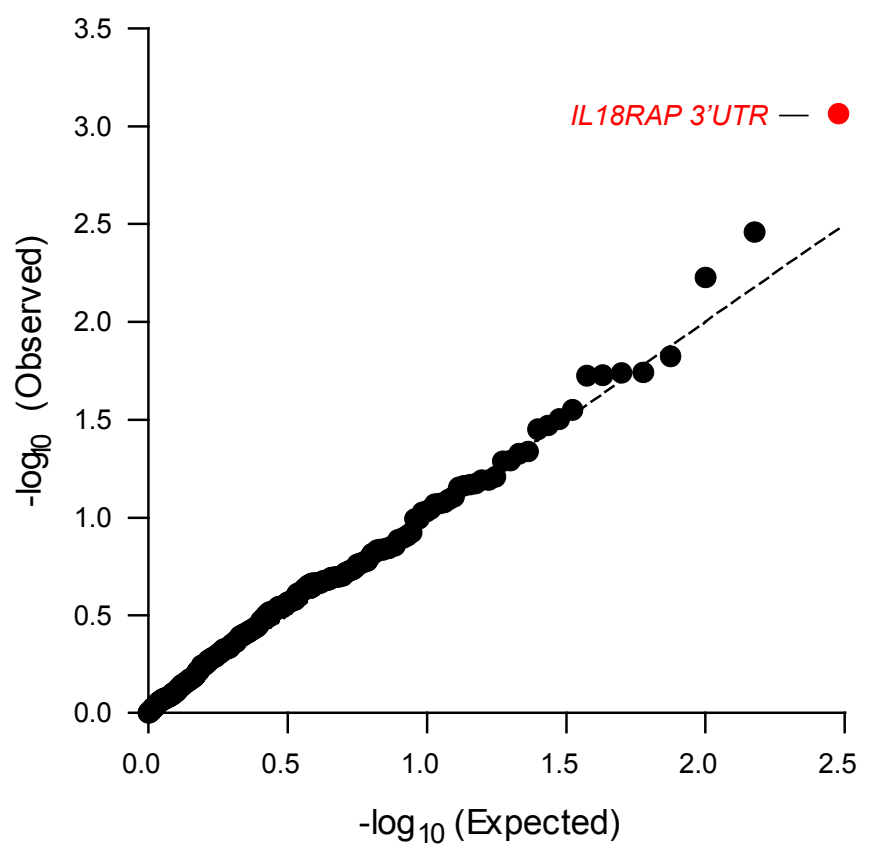

D

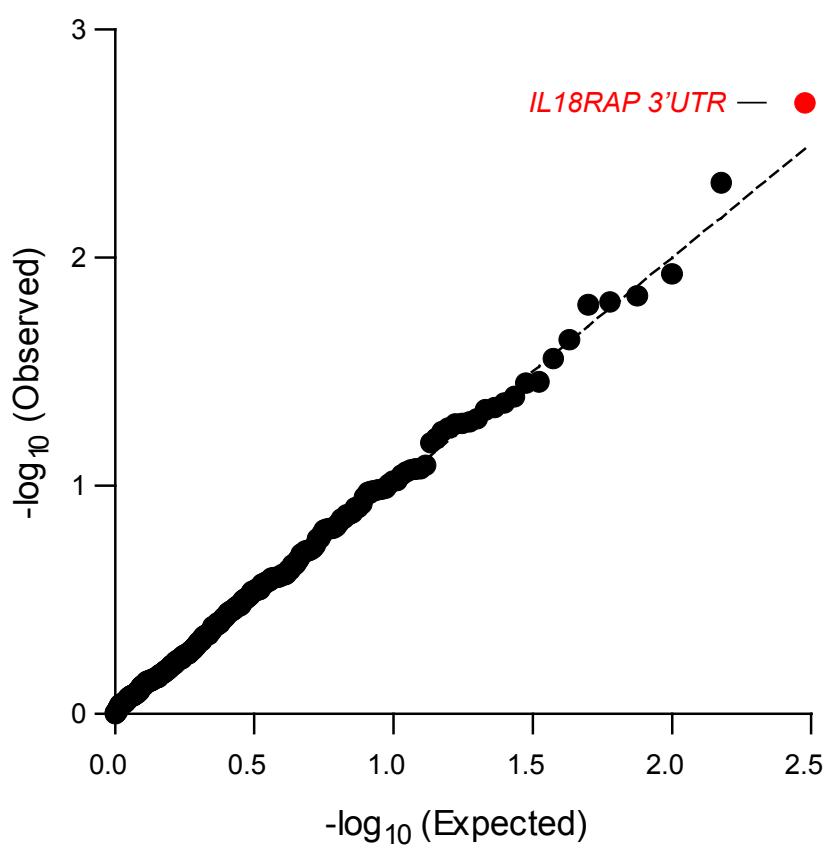

\section{Supplementary Fig.3 - Eitan et al. (Hornstein)}


698 Supplementary Fig. 3. 3'UTR-based rare-variant association analysis, using different algorithms.

699 (A-D) QQ plot of obtained and expected P-values for the burden of rare variants (log scale) gained by 700 collapsed region-based association analysis of genomic regions comprised of 295 3'UTRs listed in 701 Supplementary Table 3, in Project MinE cohort (3,955 ALS cases and 1,819 non-ALS controls). Variants 702 are not restricted to miRNA recognition elements. Features positioned on the diagonal line represent 703 results obtained under the null hypothesis. ILI8RAP 3'UTR (red) is the most significant 3'UTR associated 704 with ALS, using different algorithms: (A) Optimized Sequence Kernel Association Test, SKAT-O 705 (genomic inflation $\lambda=0.98$ ), (B) Sequence Kernel Association Test, SKAT (genomic inflation $\lambda=0.98$ ), 706 (C) Combined Multivariate and Collapsing, CMC (genomic inflation $\lambda=1.27$ ), (D) Variable Threshold 707 with permutation analysis, VT (genomic inflation $\lambda=1.07$ ). 
A

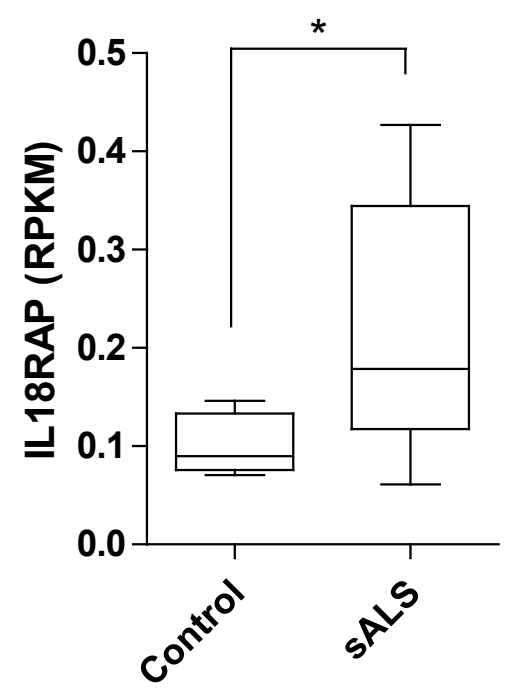

B

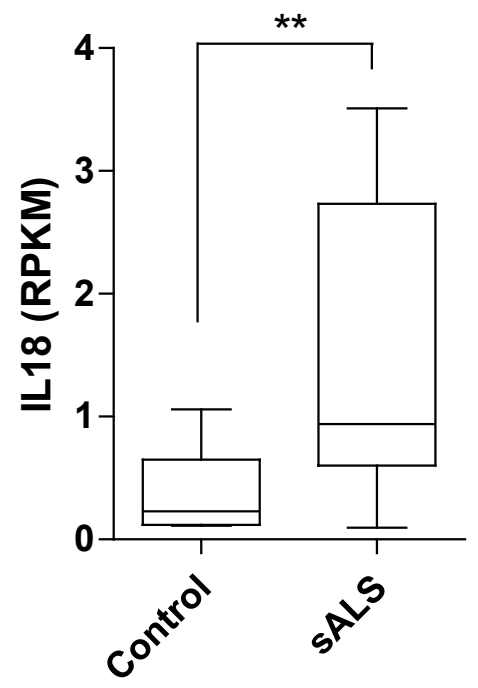

C

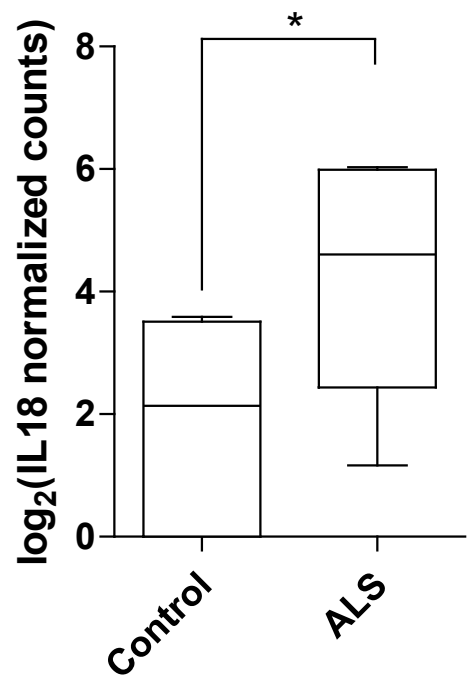


708 Supplementary Fig. 4. Evaluation of IL18RAP and IL-18 mRNA expression in motor neurons of 709 patients with ALS. (A-B) mRNA expression of IL18RAP (A) and IL-18 (B), as reads per kilobase million 710 (RPKM), from NGS study of laser capture microdissection-enriched surviving motor neurons from 711 lumbar spinal cords of patients with sALS with rostral onset and caudal progression $(\mathrm{n}=12)$ and non712 neurodegeneration controls $\left(\mathrm{n}=9 ;{ }^{43}\right.$ GSE76220). two-sided Student's $\mathrm{t}$ test with Welch's correction on 713 log-transformed data. (C) IL-18 mRNA expression, as log2-normalized counts, from NGS study of 714 induced ALS motor neurons ( $\mathrm{n}=4$ different donors in duplicates) or non-neurodegeneration controls ( $\mathrm{n}=3$ 715 different donors in duplicates; ${ }^{44}$ DESeq analysis). Box plots depicting median, upper and lower quartiles, 716 and extreme points. $* \mathrm{P}<0.05 ; * * \mathrm{P}<0.01$. 